\title{
Steady fields of coherent synchrotron radiation in a rectangular pipe
}

\author{
Tomonori Agoh \\ Tsukuba, Japan \\ (Received 22 April 2009; published 23 September 2009)
}

\begin{abstract}
We study longitudinal fields of coherent synchrotron radiation in a perfectly conducting rectangular pipe. Our theory is based on the paraxial approximation of electromagnetic waves in the frequency domain. The longitudinal impedance of coherent radiation is obtained. By considering the pole structure of the impedance in a rectangular pipe, we have derived the analytical expression of the longitudinal field in the time domain. According to the analysis, we show how the sidewalls of the vacuum chamber affect the longitudinal field of coherent radiation. In addition, we discuss the limit of applicability of the paraxial approximation.
\end{abstract}

DOI: $10.1103 /$ PhysRevSTAB.12.094402

PACS numbers: 41.75.Ht, 29.27.Bd, 41.60.Ap, 02.30.Mv

\section{INTRODUCTION}

In this paper we investigate the electromagnetic fields of coherent synchrotron radiation in electron accelerators. In high energy accelerators, when an electron bunch travels in a dipole magnet, it is bent and emits synchrotron radiation which has a wide frequency spectrum from the microwave to the $\mathrm{x}$-ray range [1]. The frequency components lower than the inverse bunch length are coherently emitted and can grow up to a huge intensity. Coherent synchrotron radiation (CSR) is referred to this low frequency region and has a typical wave number:

$$
k \sim \frac{1}{\sigma_{z}},
$$

where $\sigma_{z}$ is the bunch length. In general, the wave number of a wave, $k$, is a more fundamental quantity than its wavelength. If a bunch has a longitudinal distribution which is not smooth but has a density fluctuation, the resulting inner structure of the bunch amounts to short bunches. Thus, the CSR will have a spectrum around the bunch spectrum which is the Fourier transform of the longitudinal charge distribution. CSR is essentially a collective effect of electrons contained in a bunch and considerably differs from incoherent synchrotron radiation which is a result of particles radiating independently from each other.

In light sources or colliders, regardless of machine type, short bunch lengths are sought to satisfy various contemporary requirements, e.g., short duration time in synchrotron light, high peak current in free electron lasers, high luminosity in colliders, and so on. In addition, high current is often simultaneously required for higher machine performance. The demand for shorter bunch and higher intensity beam may persist. However, CSR would nullify the benefits of these future machines through its harmful effects on bunches. The longitudinal force induced by CSR gives rise to energy changes, which results in an energy spread of the bunch, a deterioration of transverse emit- tance, microwave instabilities and other nuisances. In any beam line having bending magnets, therefore, short bunches are under the threat of CSR.

Corresponding to progress of accelerator performance, CSR has become a serious concern in beam dynamics; a number of theories on CSR have been developed including numerical approaches. Let us introduce the past relevant studies featuring mainly analytical work. Warnock and Morton studied the radiation fields driven by a circulating beam in a toroidal chamber [2]. They discussed the resonance structure of the field and the resistive wall effects via impedance analysis. When the radiation has a phase velocity equal to the speed of the particles in a toroidal chamber, the field resonances occur due to the synchronous coupling between the field and the particles. Since the electromagnetic field of CSR is proportional to the bunch charge, the radiated power of CSR is proportional to its square. This property allows us to normalize the field by the bunch current, CSR can be parametrized using geometrical quantities of the accelerator: size of vacuum chamber, bending radius, and length of magnets. This is the virtue of the impedance representation. $\mathrm{Ng}$ dealt, around the same time, with a similar problem, which was applied to the impedance estimation for the superconducting super collider booster rings and the Tevatron [3]. In the future proton machines if the Lorentz factor is large and the bunch length is short, proton bunches will also be affected by CSR.

Warnock pursued his study on the parallel plates impedance. It was shown that frequency components below a certain value are strongly suppressed by the metallic plates; therefore the intensity is weak [4]. The criterion is called the shielding threshold, and the wave number is given by

$$
\frac{k_{\mathrm{th}}}{\pi}=\sqrt{\frac{\rho}{h^{3}}},
$$

where $\rho$ is the bending radius of the dipole magnet, and $h$ is the distance between the plates [several definitions of the coefficients of Eq. (2) are found in the literature]. Equa- 
tion (2) involves the bending radius $\rho$ and is different from the so-called cutoff frequency in a straight waveguide. Murphy et al. derived the CSR wakefield in the time domain, which is shielded by infinite parallel plates represented by image charge fields [5]. This wakefield is steady and has a periodic boundary condition along a circular orbit as in the theory of Warnock-Morton and $\mathrm{Ng}$. To accelerators, the vacuum chamber not only creates a vacuum for the beam channel but also restrains CSR emission. Therefore, an electron storage ring on service would not work on the moon in the absence of a vacuum chamber because of the huge amount of energy loss due to CSR.

After a bunch has traveled a distance from the entrance of a magnet, the CSR stops growing and becomes stationary with respect to the bunch. Assuming an infinitely long constant bending magnet in free space, Derbenev et al. derived the longitudinal and transverse forces of steady CSR [6,7]. The path length used as a criterion for steady radiation is called the overtaking length (formation length): $s_{0}=2\left(3 \rho^{2} \sigma_{z}\right)^{1 / 3}$. By considering geometry around the magnet edge with a two particle model, Saldin et al. derived the energy change due to transient CSR in free space [8]. Beside the edge of the magnet, the CSR field is transient if the charge distribution is changing as in a bunch compressor. Mayes and Hoffstaetter have developed a time domain method [9] which derives the transient wakefield of CSR between perfectly conducting parallel plates. Because of the time domain approach, one can readily consider the dynamic change of the longitudinal charge distribution. In the present paper we will examine the condition in which the parallel plates model can be a good approximation for the beam pipe.

Stupakov and Kotelnikov introduced a paraxial approximation to CSR analysis, so that Maxwell equations are fairly simplified in spite of curvilinear coordinates [10]. Considering steady field propagating in a perfectly conducting curved waveguide, they investigated the synchronous modes and the radiated power. It is noteworthy that they gave a derivation of the well-known power spectrum of synchrotron radiation [1] in the high frequency limit of their formalism. Applying the paraxial approximation, a numerical approach was developed for CSR analysis [11,12], which uses a parabolic equation of Schrödinger type. One can then obtain transient fields of CSR in a resistive pipe. With the parabolic equation, one can calculate the CSR field propagating in arbitrary combinations of drift spaces of finite length and bending magnets. The paraxial approximation was originally developed in optics where the electromagnetic wave has a high directivity and propagates nearly parallel to an optical axis. CSR is emitted with a small opening angle whose typical spread is $\vartheta \sim$ $(k \rho)^{-1 / 3}$ in free space or $\vartheta \sim \pi / k h$ between parallel plates [5]. Therefore if $\sigma_{z} \ll \rho$ in free space, or $\sigma_{z} \ll h / \pi$ between parallel plates, one can regard CSR as a paraxial ray.
Generally speaking, by including numerical calculations, the time domain approaches can be adapted to a case when the charge distribution is changing in a short time due to the bunch compression or the self-induced CSR. One can consider the effect also in the frequency domain approach by decomposing the field into Green functions; however, the time domain approach has advantages in this respect. On the other hand, as CSR is an electromagnetic wave, the frequency domain approach is rather suitable to understand fundamental properties of CSR. Besides that, it is easy to impose a resistive boundary in the frequency domain. It is also possible in the time domain to consider a resistive wall effect, because one can simply add the CSR field in the perfectly conducting pipe and the resistive wall wakefield which have been calculated separately [12]. Thus both the time domain and frequency domain approaches have their own merits, as it is stated also in [5]; they are always complementary. Other than in the literature we introduced above, there are many important works on CSR as well as incoherent radiation not covered here [13]. It is true that classical electromagnetic radiation has been studied over a century, but CSR study is still a nascent field of research; the theory and experiment including applications for light source are in progress now, and their importance may increase in the future.

This paper presents a theory of radiation based on the paraxial approximation, in which the longitudinal field of CSR is derived in the time domain, where we assume steady fields radiated in an infinitely long rectangular beam pipe. In Sec. II the field equation in the frequency domain is introduced with our coordinates and chamber model. Then we solve it analytically and obtain the electric field of CSR in the frequency domain. Section III presents the longitudinal impedance of CSR in a rectangular pipe, we show that the impedance reduces to the pillbox chamber and infinite parallel plates models in the appropriate limits. In addition, it is shown that the paraxial approximation is actually applicable to CSR even in free space if $\sigma_{z} \ll \rho$. We discuss also the shielding effect by a rectangular pipe in Sec. III, where we examine validity of the infinite parallel plates in the CSR analysis. In Sec. IV we investigate the pole structure of the impedance in the rectangular pipe, then in Sec. V we finally obtain the time domain field which is compared with our grid simulation [11]. In Sec. VI we discuss the application limit of the paraxial approximation via conversion of the real impedance from a rectangular pipe to parallel plates. We summarize our work in Sec. VII.

Our formalism is similar to Warnock-Morton's theory [2]; in fact, our impedances as shown in Sec. III can also be obtained as a limit of their expressions. We briefly introduce their work and describe the connection to ours in Appendix A, where the error by the paraxial approximation is estimated. In Appendix B we describe the asymptotic 
expansion of the impedance in the low frequency limit, which is needed in Sec. III to quantify the shielding effect by a rectangular waveguide. The asymptotic expression in the low frequency limit is also necessary to examine the regularity of the impedance around the origin of the wave number. In the analysis of the pole structure of CSR impedance, we are faced with a multivalue problem on the impedance variables. We write down the treatment for the multivalued variables in Appendix C.

\section{FIELDS IN THE FREQUENCY DOMAIN}

\section{A. Coordinates and assumptions}

We employ a coordinate system $(x, y, z ; s)$ that is often used in accelerator physics. Assuming a reference orbit, the independent variable $s$ is defined by the length along the reference orbit; $x$ and $y$ are, respectively, the horizontal and the vertical coordinates perpendicular to $s . z$ is a longitudinal variable defined by

$$
z=s-\beta t,
$$

where $\beta$ is the speed of the electrons. We assume a planar orbit in the $(x, s)$ plane with a constant curvature radius $\rho$ as shown in Fig. 1. We use SI units and set the speed of light to unity $(c=1)$ for simplicity.

To develop our theory, we assume the following things.

(a) The dipole magnet is infinitely long; the reference axis $s$ has a constant curvature radius $\rho$.

(b) The beam pipe is perfectly conducting and has a rectangular cross section of width $w$ and height $h$. The pipe cross section is uniform along the orbit.

(c) The wave number of the field $k$ satisfies

$$
k \gg \frac{\pi}{\min [w, h, \rho]} .
$$

$\pi / w$ and $\pi / h$ are the transverse cutoff wave numbers of the straight rectangular waveguide. It follows that the

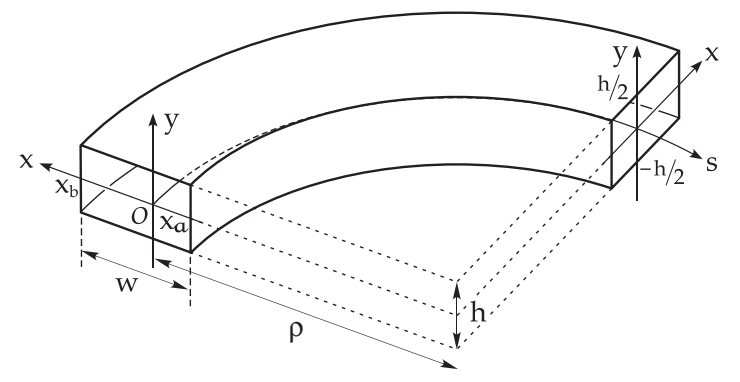

FIG. 1. Coordinate system and vacuum chamber. The reference axis $s$ lays on the median horizontal plane $(y=0)$ with a constant radius $\rho$; the horizontal axis $x$ points outwardly. The pipe has a uniform cross section and infinite length along the reference orbit. The inner and outer wall positions are $x_{a}$ and $x_{b}$, respectively, the pipe width is given by $w=x_{b}-x_{a}$, where $x_{a}<0<x_{b}$. The pipe height is $h$; the upper and lower walls are located at $y= \pm h / 2$, respectively. radiation components propagating at large angles with respect to the reference axis are negligible (paraxial approximation).

(d) The bunch consists of relativistic electrons: $\gamma \gg 1$, where $\gamma$ is the Lorentz factor and is held constant. All electrons within the bunch are assumed to have the same energy and the same velocity $\beta$. In deriving the time domain field, we assume $\gamma=\infty$.

(e) The charge distribution of the bunch $\tilde{J}_{0}(x, y, z)$ is rigid and has no internal correlation. $\tilde{J}_{0}$ has vertical symmetry with respect to the midhorizontal plane. To obtain the impedance and the time domain field, moreover, the bunch is assumed to have no horizontal dimension.

By the assumptions (a), (b), and (e), the field is steady. We may need to mention a subtle problem in our assumption. Because of condition (d), rigorously speaking, a rigid bunch cannot have a finite horizontal width. Denoting the width as $\sigma_{x}$, however, the error caused by the contradiction is of the order of $\sigma_{x} / \rho$ in the longitudinal field, unless the particles exceed the speed of light.

Let $k$ and $\omega$ be the wave number and the frequency of the field; the phase velocity is given by $\omega / k$. Since we are assuming that all particles travel at the same speed of $\beta$, the radiation which interacts with the particles has the same phase velocity as the particles: $\omega=\beta k$. Therefore, it is enough to consider a Fourier transform only with respect to the wave number, i.e.,

$$
\begin{gathered}
\tilde{f}(z)=\frac{1}{2 \pi} \int_{-\infty}^{\infty} f(k) e^{i k z} d k, \\
f(k)=\int_{-\infty}^{\infty} \tilde{f}(z) e^{-i k z} d z .
\end{gathered}
$$

Since we mainly work in the frequency domain, we put tilde on top of the time domain variables as $\tilde{\boldsymbol{E}}(z), \tilde{\lambda}(z)$. On the other hand, variables without the tilde are quantities in the frequency domain. Equations (5) and (6) imply that we do not impose periodic boundary conditions for the field along the beam orbit.

\section{B. Field equation in the paraxial approximation}

Under the assumptions (c) and (d), Maxwell equations in the frequency domain reduce to the following form [12]:

$$
2 i k \frac{\partial \boldsymbol{E}_{\perp}}{\partial s}+\left\{\boldsymbol{\nabla}_{\perp}^{2}+2 k^{2}\left(\frac{x}{\rho}-\frac{1}{2 \gamma^{2}}\right)\right\} \boldsymbol{E}_{\perp}=\mu_{0} \boldsymbol{\nabla}_{\perp} J_{0},
$$

where $\boldsymbol{E}_{\perp}$ is the transverse electric field: $\boldsymbol{E}_{\perp}=\left(E_{x}, E_{y}\right)$, $\boldsymbol{\nabla}_{\perp}$ is the transverse gradient: $\boldsymbol{\nabla}_{\perp}=\left(\partial_{x}, \partial_{y}\right), \mu_{0}$ is the permeability of vacuum. In Eq. (7) the leading term we neglected is of the order of $(k \rho)^{-2 / 3} . J_{0}$ on the right-hand side of Eq. (7) is the charge distribution of the bunch in the frequency domain,

$$
J_{0}(x, y, k)=q_{e} \lambda(k) \Psi_{x}(x) \Psi_{y}(y) .
$$

$q_{e}$ is the bunch charge, and $\Psi_{x}(x), \Psi_{y}(y)$ are, respectively, 
the horizontal and the vertical charge distributions of the bunch. $\lambda(k)$ is the bunch spectrum which is the Fourier transform of the longitudinal charge distribution $\tilde{\lambda}(z)$ :

$$
\lambda(k)=\int_{-\infty}^{\infty} \tilde{\lambda}(z) e^{-i k z} d z .
$$

$\tilde{\lambda}(z), \Psi_{x}(x)$, and $\Psi_{y}(y)$ are normalized to unity.

If the field is in a steady state, the transverse electric field satisfies

$$
\left\{\nabla_{\perp}^{2}+2 k^{2}\left(\frac{x}{\rho}-\frac{1}{2 \gamma^{2}}\right)\right\} \boldsymbol{E}_{\perp}=\mu_{0} \boldsymbol{\nabla}_{\perp} J_{0} .
$$

At high energy, the field slightly depends on the energy of the particles, as Eq. (10) shows, the finite energy is equivalent to a horizontal displacement given by $\delta x=\rho / 2 \gamma^{2}$. The longitudinal field is obtained by the transverse field through Gauss's law and Faraday's law,

$$
\begin{gathered}
E_{s}=\frac{i}{k}\left(\nabla_{\perp} \cdot E_{\perp}-\mu_{0} J_{0}\right), \\
B_{s}=\frac{i}{k}\left(\partial_{y} E_{x}-\partial_{x} E_{y}\right) .
\end{gathered}
$$

From these equations, the transverse Lorentz force per unit charge is obtained:

$$
\begin{aligned}
& F_{x}=+\frac{i}{2 k}\left(\partial_{y} B_{s}-\partial_{x} E_{s}\right), \\
& F_{y}=-\frac{i}{2 k}\left(\partial_{x} B_{s}+\partial_{y} E_{s}\right) .
\end{aligned}
$$

However, we do not deal with the transverse force in this paper; our work is strictly devoted to analysis of the longitudinal electric field and its impedance. Let us give a comment on the transverse force by CSR. The vertical force has a similar nature to $E_{s}$, i.e., $F_{y}$ hardly depends on $\Psi_{x}$ and $\Psi_{y}[7,12]$. On the other hand, the horizontal force may strongly depend on the horizontal charge distribution. For the future development of the transverse force, we will keep $\Psi_{x}$ in the frequency domain field.

We define a variable $\kappa$ as

$$
\kappa=\left(\frac{2 k^{2}}{\rho}\right)^{1 / 3},
$$

which is a function of the wave number and has dimension of inverse length. For a given wave number, $\kappa^{-1}$ has a typical scale length of the transverse expanse of the field in free space; therefore, if we put $\kappa h=1$ and solve it with respect to the wave number, we obtain $k=\left(\rho / 2 h^{3}\right)^{1 / 2}$ which is approximately equal to the threshold wave number of Eq. (2). We normalize the transverse coordinates $(x, y)$ with $\kappa$ into the dimensionless variables:

$$
\xi=\kappa x, \quad \eta=\kappa y .
$$

Similarly, we normalize the sidewalls position with $\kappa$ :

$$
\xi_{a}=\kappa x_{a}, \quad \xi_{b}=\kappa x_{b} .
$$

With the dimensionless transverse coordinates, we define the horizontal and the vertical charge distribution which are normalized to unity for $\xi, \eta$, respectively:

$$
\psi_{x}(\xi)=\frac{\Psi_{x}(x)}{\kappa}, \quad \psi_{y}(\eta)=\frac{\Psi_{y}(y)}{\kappa} .
$$

We expand the electric field and the vertical charge distribution in vertical Fourier series:

$$
\begin{gathered}
\boldsymbol{E}(\xi, \eta)=\sum_{n=-\infty}^{\infty} \overrightarrow{\mathcal{E}}^{n}(\xi) e^{i \varphi_{n}}, \\
\psi_{y}(\eta)=\sum_{n=-\infty}^{\infty} \psi_{y}^{n} e^{i \varphi_{n}}, \\
\varphi_{n}=\frac{\pi \eta}{\kappa h} n
\end{gathered}
$$

where $\overrightarrow{\mathcal{E}}^{n}=\left(\mathcal{E}_{x}^{n}, \mathcal{E}_{y}^{n}, \mathcal{E}_{s}^{n}\right)$ and $\psi_{y}^{n}$ are, respectively, the Fourier coefficients of the field and the vertical charge distribution of the bunch. We assume that the charge distribution is symmetric in the vertical direction, $\psi_{y}(-\eta)=\psi_{y}(\eta)$, and then the field components must be symmetric or antisymmetric with respect to the median horizontal plane and reduce to the following form:

$$
\begin{gathered}
\boldsymbol{E}(\xi, \eta)=\overrightarrow{\mathcal{E}}^{0}+2 \sum_{n=1}^{\infty}\left(\begin{array}{c}
\mathcal{E}_{x}^{n} \cos \varphi_{n} \\
i \mathcal{E}_{y}^{n} \sin \varphi_{n} \\
\mathcal{E}_{s}^{n} \cos \varphi_{n}
\end{array}\right), \\
\psi_{y}(\eta)=\psi_{y}^{0}+\sum_{n=1}^{\infty} 2 \psi_{y}^{n} \cos \varphi_{n} .
\end{gathered}
$$

In the perfectly conducting pipe, the transverse field satisfies the following boundary condition on the upper-lower walls for any $\xi$ in the pipe: $\xi_{a}<\xi<\xi_{b}$ :

$$
\begin{gathered}
\left.E_{x}\right|_{y= \pm h / 2}=0, \\
\left.\partial_{y} E_{y}\right|_{y= \pm h / 2}=0 .
\end{gathered}
$$

Therefore, the constant terms in the Fourier series are zero: $\overrightarrow{\mathcal{E}}^{0}=0, \psi_{y}^{0}=0$. The boundary condition on the upperlower walls constrains the vertical Fourier modes into $n=$ $2 p+1(p=0,1,2,3, \ldots)$. Thus only the odd modes can propagate in the chamber; because the beam drives the field, its position cannot be a node of $E_{x}$ and $E_{s}$ which form standing waves in the vertical direction. If the beam deviates off the midhorizontal plane, then the even modes are excited. However, we do not treat this case in this paper.

We define the vertical wave number of the field by $\alpha_{p}$ :

$$
\alpha_{p}=\frac{\pi}{h}(2 p+1) .
$$

We often use the dimensionless vertical wave number $\beta_{p}$ 
instead of $\alpha_{p}$, defined as

$$
\beta_{p}=\frac{\alpha_{p}}{\kappa},
$$

which depends on $k$ through $\kappa$. For simpler notation, we redefine the superscript of the vertical mode index: $\overrightarrow{\mathcal{E}}^{n}=\overrightarrow{\mathcal{E}}^{2 p+1}$ as $\overrightarrow{\mathcal{E}}^{p}$, and $\psi_{y}^{n}=\psi_{y}^{2 p+1}$ as $\psi_{y}^{p}$, respectively. Because of the perfectly conducting rectangular pipe, we can separately deal with the vertical mode of the field. The normalized field equation for the vertical mode $p$ is

$$
\left(\partial_{\xi}^{2}+\xi-w_{p}\right) \mathcal{E}_{\perp}^{p}=I_{\perp}^{p} .
$$

$\mathcal{E}_{\perp}^{p}=\left(\mathcal{E}_{x}^{p}, \mathcal{E}_{y}^{p}\right)$ is the transverse field. $w_{p}$ is given by

$$
w_{p}=\beta_{p}^{2}+\frac{\kappa \rho}{2 \gamma^{2}} \text {. }
$$

$I_{\perp}^{p}=\left(I_{x}^{p}, I_{y}^{p}\right)$ on the right-hand side of Eq. (27) is the driving term of the field,

$$
I_{\perp}^{p}=\mu_{0} \kappa q_{e} \lambda(k)\left(\begin{array}{c}
\partial_{\xi} \\
i \beta_{p}
\end{array}\right) \psi_{x}(\xi) \psi_{y}^{p} .
$$

Having solved Eq. (27), the longitudinal field is obtained in terms of the transverse field via

$$
\mathcal{E}_{s}^{p}=\frac{i \kappa}{k}\left(\partial_{\xi} \mathcal{E}_{x}^{p}+i \beta_{p} \mathcal{E}_{y}^{p}-\mu_{0} \kappa q_{e} \lambda \psi_{x} \psi_{y}^{p}\right) .
$$

The vertical Fourier mode of the vertical charge distribution is

$$
\psi_{y}^{p}=\frac{1}{\kappa h} \int_{-\kappa h / 2}^{\kappa h / 2} \psi_{y}(\eta) e^{i \beta_{p} \eta} d \eta .
$$

The transverse distribution of the bunch, $\psi_{x}$ and $\psi_{y}$, is usually not important to the longitudinal field of CSR for high energy particles, but it is necessary later when we consider the space charge effect. Meanwhile, if one calculates the horizontal force of Eq. (13a), the horizontal charge distribution $\psi_{x}$ should have a finite width.

\section{Solution of the field equation}

To solve Eq. (27), we will use the technique of variation of parameters. Therefore we first solve the homogeneous version. If Eq. (27) is homogeneous, it reduces to Eqs. (17) of [10] and satisfies the Airy differential equation:

$$
\frac{d^{2} f(r)}{d r^{2}}=r f(r)
$$

where $r$ is the variable of the Airy function and can be complex in general. In our problem,

$$
r=w_{p}-\xi
$$

The Airy differential equation has three pairs of linearly independent fundamental solutions which damp at infinity in some direction on the complex $r$ plane [14]:
(1) $\operatorname{Ai}(r)$ and $\operatorname{Bi}(r)$,
(2) $\mathrm{Ai}(r)$ and $\operatorname{Ci}(r) \equiv \operatorname{Ai}(r)-i \operatorname{Bi}(r)$,
(3) $\operatorname{Ai}(r)$ and $\operatorname{Di}(r) \equiv \operatorname{Ai}(r)+i \operatorname{Bi}(r)$.

In the limit $|r| \rightarrow \infty, \mathrm{Ci}(r)$ is bounded on the arguments $\arg r=\pi / 3$ and $-2 \pi / 3$; on the other hand, $\operatorname{Di}(r)$ is bounded on $\arg r=-\pi / 3$ and $2 \pi / 3$.

We will specify a pair of the solutions to describe the radiation field in our problem. Let us examine the asymptotic behavior of the solutions $\mathrm{Ai}(r), \mathrm{Bi}(r)$ for positive large $r$ :

$$
\begin{aligned}
& \mathrm{Ai}(r) \simeq \frac{r^{-1 / 4}}{2 \sqrt{\pi}} e^{-\zeta_{r}}, \\
& \mathrm{Bi}(r) \simeq \frac{r^{-1 / 4}}{\sqrt{\pi}} e^{+\zeta_{r},} \\
& \zeta_{r}=(2 / 3) r^{3 / 2} \in \mathbb{R} .
\end{aligned}
$$

The Airy function $\operatorname{Ai}(r)=\operatorname{Ai}\left(w_{p}-\xi\right)$ exponentially goes to zero for $r \rightarrow+\infty$ (i.e., inside the orbit $\xi \rightarrow-\infty$ ) and agrees with the behavior of CSR inside the orbit because the radiation is emitted tangent to the orbit with a small opening angle, the field should rapidly diminish inside the orbit. In contrast, $\mathrm{Bi}\left(w_{p}-\xi\right)$ exponentially diverges inside the orbit: $r \rightarrow+\infty(\xi \rightarrow-\infty)$ and thereby cannot represent field components.

Next, we seek a solution which meets the outward field of CSR. Outside the orbit, i.e., $r \rightarrow-\infty(\xi \rightarrow+\infty)$, the Airy functions, $\mathrm{Ci}$ and $\mathrm{Di}$, behave as

$$
\begin{aligned}
\operatorname{Ci}(r) & \simeq \frac{(-r)^{-1 / 4}}{\sqrt{\pi}} e^{+i\left(\zeta_{r}^{\prime}-\pi / 4\right)}, \\
\operatorname{Di}(r) & \simeq \frac{(-r)^{-1 / 4}}{\sqrt{\pi}} e^{-i\left(\zeta_{r}^{\prime}-\pi / 4\right)}, \\
\zeta_{r}^{\prime} & =(2 / 3)(-r)^{3 / 2} \in \mathbb{R} .
\end{aligned}
$$

Since our Fourier transform is defined by Eq. (5), the time domain field involves $e^{-i \omega t}$. Therefore the Fourier counterparts of $\mathrm{Ci}\left(w_{p}-\xi\right)$ and $\operatorname{Di}\left(w_{p}-\xi\right)$ outside the orbit are roughly described as

$$
\begin{aligned}
& \mathcal{F}\left[\operatorname{Ci}\left(w_{p}-\xi\right)\right] \propto e^{+i\left(\zeta_{r}^{\prime}-\omega t\right)}, \\
& \mathcal{F}\left[\operatorname{Di}\left(w_{p}-\xi\right)\right] \propto e^{-i\left(\zeta_{r}^{\prime}+\omega t\right)} .
\end{aligned}
$$

The symbol $\mathcal{F}$ indicates the longitudinal Fourier transform. From Eqs. (41) and (42), $\mathrm{Ci}\left(w_{p}-\xi\right)$ can be identified with the outwardly propagating field, while $\operatorname{Di}\left(w_{p}-\xi\right)$ behaves as if the radiation is inwardly emitted and so is irrelevant. Thus, it turned out that the pair of the solutions given by Eq. (34b) fulfills the property of CSR. It is therefore natural to give the transverse field by a linear 
combination of $\mathrm{Ai}$ and $\mathrm{Ci}$ :

$$
\frac{\mathcal{E}_{\perp}^{p}(\xi)}{i \pi}=a_{\perp}^{p} \operatorname{Ai}\left(w_{p}-\xi\right)+c_{\perp}^{p} \operatorname{Ci}\left(w_{p}-\xi\right)
$$

where the factor $i \pi$ has no physical sense but is introduced for convenience in later calculations. $a_{\perp}^{p}, c_{\perp}^{p}$ are coefficients of the linear combinations; they will depend on $\xi$ in our problem [Eq. (44)]. Another pair $\operatorname{Ai}(r)$ and $\mathrm{Bi}(r)$ is, of course, available to describe the CSR field, because $\mathrm{Ci}$ is a linear combination of $\mathrm{Ai}$ and $\mathrm{Bi}$. If using the real notation (Ai, Bi), however, the following calculations will be somewhat complicated. Besides that, the complex notation (Ai, $\mathrm{Ci}$ ) corresponds to the inward/outward field of CSR and provides a clear physical picture.

The variation of parameters on Eq. (43) gives

$$
\begin{aligned}
& a_{\perp}^{p}(\xi)=a_{0 \perp}^{p}-\int_{\xi}^{\xi_{b}} I_{\perp}^{p}\left(\xi^{\prime}\right) \operatorname{Ci}\left(w_{p}-\xi^{\prime}\right) d \xi^{\prime}, \\
& c_{\perp}^{p}(\xi)=c_{0 \perp}^{p}-\int_{\xi_{a}}^{\xi} I_{\perp}^{p}\left(\xi^{\prime}\right) \operatorname{Ai}\left(w_{p}-\xi^{\prime}\right) d \xi^{\prime},
\end{aligned}
$$

where $a_{0 \perp}^{p}$ and $c_{0 \perp}^{p}$ are the constants of integration. The boundary condition on the sidewalls is

$$
\begin{gathered}
\left.\partial_{x} E_{x}\right|_{x=x_{a}, x_{b}}=0, \\
\left.E_{y}\right|_{x=x_{a}, x_{b}}=0,
\end{gathered}
$$

which fix the values of $a_{0 \perp}^{p}$ and $c_{0 \perp}^{p}$. For a simple notation, we define the arguments of the Airy functions:

$$
\begin{aligned}
& u_{p}=w_{p}-\xi_{a}, \\
& v_{p}=w_{p}-\xi_{b}, \\
& w_{\xi}=w_{p}-\xi,
\end{aligned}
$$

and $w_{p}$ is given by Eq. (28). $u_{p}, v_{p}$ are variables that are associated with the inner and the outer wall, respectively. In accordance with the notation of $[2,14]$, we define the cross products of the Airy functions as follows:

$$
\begin{aligned}
& \hat{p}(x, y)=\operatorname{Ai}(x) \operatorname{Ci}(y)-\operatorname{Ci}(x) \operatorname{Ai}(y), \\
& \hat{q}(x, y)=\operatorname{Ai}(x) \mathrm{Ci}^{\prime}(y)-\operatorname{Ci}(x) \operatorname{Ai}^{\prime}(y), \\
& \hat{r}(x, y)=\operatorname{Ai}^{\prime}(x) \mathrm{Ci}(y)-\mathrm{Ci}^{\prime}(x) \operatorname{Ai}(y), \\
& \hat{s}(x, y)=\operatorname{Ai}^{\prime}(x) \operatorname{Ci}^{\prime}(y)-\operatorname{Ci}^{\prime}(x) \operatorname{Ai}^{\prime}(y),
\end{aligned}
$$

where $x, y$ are not the transverse coordinates but arbitrary complex variables, and the primes denote the derivatives. $\hat{q}(x, x)$ is the Wronskian of $\mathrm{Ai}$ and $\mathrm{Ci}$ :

$$
\hat{q}(x, x)=\frac{1}{i \pi}
$$

With these symbols, the horizontal field in the vertical Fourier mode is given by

$$
\begin{aligned}
\frac{\mathcal{E}_{x}^{p}(\xi)}{A}= & -\operatorname{Ci}\left(w_{\xi}\right) \int_{\xi_{a}}^{\xi} \psi_{x}\left(\xi^{\prime}\right) \operatorname{Ai}^{\prime}\left(w_{\xi^{\prime}}\right) d \xi^{\prime}+\frac{\hat{q}\left(w_{\xi}, u_{p}\right)}{\hat{s}\left(v_{p}, u_{p}\right)} \operatorname{Ci}^{\prime}\left(v_{p}\right) \int_{\xi_{a}}^{\xi_{b}} \psi_{x}\left(\xi^{\prime}\right) \operatorname{Ai}^{\prime}\left(w_{\xi^{\prime}}\right) d \xi^{\prime} \\
& -\operatorname{Ai}\left(w_{\xi}\right) \int_{\xi}^{\xi_{b}} \psi_{x}\left(\xi^{\prime}\right) \operatorname{Ci}^{\prime}\left(w_{\xi^{\prime}}\right) d \xi^{\prime}+\frac{\hat{r}\left(v_{p}, w_{\xi}\right)}{\hat{s}\left(v_{p}, u_{p}\right)} \operatorname{Ai}^{\prime}\left(u_{p}\right) \int_{\xi_{a}}^{\xi_{b}} \psi_{x}\left(\xi^{\prime}\right) \operatorname{Ci}^{\prime}\left(w_{\xi^{\prime}}\right) d \xi^{\prime} .
\end{aligned}
$$

The coefficient $A$ on the left-hand side is $A=i \pi \mu_{0} \kappa q_{e} \lambda \psi_{y}^{p}$. Similarly, the vertical field can be obtained as follows:

$$
\begin{aligned}
\frac{\mathcal{E}_{y}^{p}(\xi)}{i \beta_{p} A}= & -\operatorname{Ci}\left(w_{\xi}\right) \int_{\xi_{a}}^{\xi} \psi_{x}\left(\xi^{\prime}\right) \operatorname{Ai}\left(w_{\xi^{\prime}}\right) d \xi^{\prime}+\frac{\hat{p}\left(w_{\xi}, u_{p}\right)}{\hat{p}\left(v_{p}, u_{p}\right)} \operatorname{Ci}\left(v_{p}\right) \int_{\xi_{a}}^{\xi_{b}} \psi_{x}\left(\xi^{\prime}\right) \operatorname{Ai}\left(w_{\xi^{\prime}}\right) d \xi^{\prime} \\
& -\operatorname{Ai}\left(w_{\xi}\right) \int_{\xi}^{\xi_{b}} \psi_{x}\left(\xi^{\prime}\right) \operatorname{Ci}\left(w_{\xi^{\prime}}\right) d \xi^{\prime}+\frac{\hat{p}\left(v_{p}, w_{\xi}\right)}{\hat{p}\left(v_{p}, u_{p}\right)} \operatorname{Ai}\left(u_{p}\right) \int_{\xi_{a}}^{\xi_{b}} \psi_{x}\left(\xi^{\prime}\right) \operatorname{Ci}\left(w_{\xi^{\prime}}\right) d \xi^{\prime}
\end{aligned}
$$

Then we obtain the longitudinal field from Eq. (30),

$$
\mathcal{E}_{s}^{p}(\xi)=A \frac{i \kappa}{k}\left(\hat{G}_{x}+\beta_{p}^{2} \hat{G}_{y}\right)
$$

where $\hat{G}_{x}$ and $\hat{G}_{y}$ are functions of the horizontal axis, the vertical wave number, and the longitudinal wave number: 


$$
\begin{aligned}
\hat{G}_{x}\left(\xi, \beta_{p}, k\right)= & \mathrm{Ci}^{\prime}\left(w_{\xi}\right) \int_{\xi_{a}}^{\xi} \psi_{x}\left(\xi^{\prime}\right) \mathrm{Ai}^{\prime}\left(w_{\xi^{\prime}}\right) d \xi^{\prime}-\frac{\hat{s}\left(w_{\xi}, u_{p}\right)}{\hat{s}\left(v_{p}, u_{p}\right)} \mathrm{Ci}^{\prime}\left(v_{p}\right) \int_{\xi_{a}}^{\xi_{b}} \psi_{x}\left(\xi^{\prime}\right) \mathrm{Ai}^{\prime}\left(w_{\xi^{\prime}}\right) d \xi^{\prime} \\
& +\operatorname{Ai}^{\prime}\left(w_{\xi}\right) \int_{\xi}^{\xi_{b}} \psi_{x}\left(\xi^{\prime}\right) \mathrm{Ci}^{\prime}\left(w_{\xi^{\prime}}\right) d \xi^{\prime}-\frac{\hat{s}\left(v_{p}, w_{\xi}\right)}{\hat{s}\left(v_{p}, u_{p}\right)} \operatorname{Ai}^{\prime}\left(u_{p}\right) \int_{\xi_{a}}^{\xi_{b}} \psi_{x}\left(\xi^{\prime}\right) \mathrm{Ci}^{\prime}\left(w_{\xi^{\prime}}\right) d \xi^{\prime}, \\
\hat{G}_{y}\left(\xi, \beta_{p}, k\right)= & \operatorname{Ci}\left(w_{\xi}\right) \int_{\xi_{a}}^{\xi} \psi_{x}\left(\xi^{\prime}\right) \operatorname{Ai}\left(w_{\xi^{\prime}}\right) d \xi^{\prime}-\frac{\hat{p}\left(w_{\xi}, u_{p}\right)}{\hat{p}\left(v_{p}, u_{p}\right)} \operatorname{Ci}\left(v_{p}\right) \int_{\xi_{a}}^{\xi_{b}} \psi_{x}\left(\xi^{\prime}\right) \operatorname{Ai}\left(w_{\xi^{\prime}}\right) d \xi^{\prime} \\
& +\operatorname{Ai}\left(w_{\xi}\right) \int_{\xi}^{\xi_{b}} \psi_{x}\left(\xi^{\prime}\right) \operatorname{Ci}\left(w_{\xi^{\prime}}\right) d \xi^{\prime}-\frac{\hat{p}\left(v_{p}, w_{\xi}\right)}{\hat{p}\left(v_{p}, u_{p}\right)} \operatorname{Ai}\left(u_{p}\right) \int_{\xi_{a}}^{\xi_{b}} \psi_{x}\left(\xi^{\prime}\right) \operatorname{Ci}\left(w_{\xi^{\prime}}\right) d \xi^{\prime}
\end{aligned}
$$

$\hat{G}_{x}, \hat{G}_{y}$ are ascribed to the horizontal and the vertical electric fields, respectively; we will call them the horizontal electric mode (HE mode) and the vertical electric mode (VE mode). These two modes, respectively, correspond to TE mode and TM mode in [2,3], $E_{r}$ mode and $E_{z}$ mode in [10].

\section{Thin beam approximation}

In modern accelerators, the transverse charge distribution of a bunch is very small compared to the typical size of the transverse expanse of the CSR field: $\kappa \sigma_{\perp} \ll 1$, the transverse beam spread $\sigma_{\perp}$ hardly varies the overtaking length of CSR. Therefore it is sufficient to assume that the transverse charge distribution is infinitely thin. But if one considers the space charge field which depends on the particles energy via $1 / \gamma^{2}$, it is necessary to consider the transverse distribution in either the horizontal or vertical direction, otherwise the space charge term diverges due to the infinite charge density at the beam position. At high energy, however, the radiation field is dominant and the longitudinal space charge force is very small and negligible. One can drop the space charge term by taking the limit $\gamma \rightarrow \infty$ for high energy particles, or one can separate the space charge field from the radiation field and handle it separately with a transverse beam size. Then the resulting thin beam approximation consequently works in the analysis of the longitudinal field of CSR. For the space charge field discussed later, we keep only the vertical distribution with a finite spread. In what follows our bunch model is a vertical ribbon.

When the beam travels on the reference axis, the horizontal charge distribution is given by

$$
\psi_{x}(\xi)=\delta(\xi)
$$

If one wants to consider a horizontal beam deviation $x_{0}=$ $\xi_{0} / \kappa$ from the reference orbit, one can merely put $\delta(\xi-$ $\left.\xi_{0}\right)$ in Eq. (57). With Eq. (57), the functions $\hat{G}_{x}, \hat{G}_{y}$ reduce to

$$
\begin{aligned}
\hat{G}_{x}= & \operatorname{Ci}^{\prime}\left(w_{p}\right)\left[\theta(-\xi) \mathrm{Ai}^{\prime}\left(w_{\xi}\right)-\frac{\hat{s}\left(v_{p}, w_{\xi}\right)}{\hat{s}\left(v_{p}, u_{p}\right)} \mathrm{Ai}^{\prime}\left(u_{p}\right)\right] \\
& +\operatorname{Ai}^{\prime}\left(w_{p}\right)\left[\theta(\xi) \mathrm{Ci}^{\prime}\left(w_{\xi}\right)-\frac{\hat{s}\left(w_{\xi}, u_{p}\right)}{\hat{s}\left(v_{p}, u_{p}\right)} \mathrm{Ci}^{\prime}\left(v_{p}\right)\right], \\
\hat{G}_{y}= & \operatorname{Ci}\left(w_{p}\right)\left[\theta(-\xi) \operatorname{Ai}\left(w_{\xi}\right)-\frac{\hat{p}\left(v_{p}, w_{\xi}\right)}{\hat{p}\left(v_{p}, u_{p}\right)} \operatorname{Ai}\left(u_{p}\right)\right] \\
& +\operatorname{Ai}\left(w_{p}\right)\left[\theta(\xi) \operatorname{Ci}\left(w_{\xi}\right)-\frac{\hat{p}\left(w_{\xi}, u_{p}\right)}{\hat{p}\left(v_{p}, u_{p}\right)} \operatorname{Ci}\left(v_{p}\right)\right],
\end{aligned}
$$

where $\theta(\xi)$ is the step function:

$$
\theta(\xi)= \begin{cases}1 & \text { for } \xi \geq 0 \\ 0 & \text { for } \xi<0\end{cases}
$$

We are interested in the coupling between the beam and the self-induced field, so we calculate the field at the beam position: $(\xi, \eta)=0 . \hat{G}_{x}$ and $\hat{G}_{y}$ at the beam position are given by

$$
\begin{aligned}
& \hat{G}_{x}=\operatorname{Ai}^{\prime}\left(w_{p}\right) \operatorname{Ci}^{\prime}\left(w_{p}\right)-\hat{H}_{x}\left(u_{p}, v_{p}, w_{p}\right), \\
& \hat{G}_{y}=\operatorname{Ai}\left(w_{p}\right) \operatorname{Ci}\left(w_{p}\right)-\hat{H}_{y}\left(u_{p}, v_{p}, w_{p}\right),
\end{aligned}
$$

where

$$
\begin{aligned}
\hat{H}_{x}= & \frac{\hat{s}\left(w_{p}, u_{p}\right)}{\hat{s}\left(v_{p}, u_{p}\right)} \mathrm{Ci}^{\prime}\left(v_{p}\right) \mathrm{Ai}^{\prime}\left(w_{p}\right) \\
& +\frac{\hat{s}\left(v_{p}, w_{p}\right)}{\hat{s}\left(v_{p}, u_{p}\right)} \operatorname{Ai}^{\prime}\left(u_{p}\right) \mathrm{Ci}^{\prime}\left(w_{p}\right), \\
\hat{H}_{y}= & \frac{\hat{p}\left(w_{p}, u_{p}\right)}{\hat{p}\left(v_{p}, u_{p}\right)} \mathrm{Ci}\left(v_{p}\right) \operatorname{Ai}\left(w_{p}\right) \\
& +\frac{\hat{p}\left(v_{p}, w_{p}\right)}{\hat{p}\left(v_{p}, u_{p}\right)} \operatorname{Ai}\left(u_{p}\right) \operatorname{Ci}\left(w_{p}\right) .
\end{aligned}
$$

The first terms on the right-hand side of Eqs. (60) do not depend on the sidewalls position and are identical to the terms in the parallel plates model [see Eq. (77)]. On the other hand, $\hat{H}_{x}$ and $\hat{H}_{y}$ are particular terms of the rectangular pipe and depend on the sidewalls positions through 
$u_{p}$ and $v_{p}$. A simple modification gives

$$
\begin{aligned}
\hat{G}_{x} & =\frac{\hat{s}\left(v_{p}, w_{p}\right) \hat{s}\left(w_{p}, u_{p}\right)}{\hat{s}\left(v_{p}, u_{p}\right)}, \\
\hat{G}_{y} & =\frac{\hat{p}\left(v_{p}, w_{p}\right) \hat{p}\left(w_{p}, u_{p}\right)}{\hat{p}\left(v_{p}, u_{p}\right)} .
\end{aligned}
$$

For the following calculations, we define another set of cross products with $\mathrm{Ai}$ and $\mathrm{Bi}$, which are, unlike the former set, real functions for real arguments,

$$
\begin{aligned}
& \check{p}(x, y)=\operatorname{Ai}(x) \operatorname{Bi}(y)-\operatorname{Bi}(x) \operatorname{Ai}(y), \\
& \check{q}(x, y)=\operatorname{Ai}(x) \operatorname{Bi}^{\prime}(y)-\operatorname{Bi}(x) \operatorname{Ai}^{\prime}(y), \\
& \check{r}(x, y)=\operatorname{Ai}^{\prime}(x) \operatorname{Bi}^{\prime}(y)-\operatorname{Bi}^{\prime}(x) \operatorname{Ai}(y), \\
& \check{s}(x, y)=\operatorname{Ai}^{\prime}(x) \operatorname{Bi}^{\prime}(y)-\operatorname{Bi}^{\prime}(x) \operatorname{Ai}^{\prime}(y) .
\end{aligned}
$$

They are related to the former cross products as follows:

$$
\check{\tau}(x, y)=i \hat{\tau}(x, y),
$$

where $\check{\tau}$ (or $\hat{\tau}$ ) represents either $\check{p}, \check{q}, \check{r}$, or $\check{s}(\hat{p}, \hat{q}, \hat{r}, \hat{s})$. $\breve{q}(x, x)$ is the Wronskian of $\mathrm{Ai}$ and $\mathrm{Bi}$ :

$$
\check{q}(x, x)=\frac{1}{\pi} .
$$

With these new cross products, we define $\check{G}_{x}$ and $\check{G}_{y}$ as

$$
\begin{aligned}
& \check{G}_{x}=i \hat{G}_{x}=\frac{\check{s}\left(v_{p}, w_{p}\right) \check{s}\left(w_{p}, u_{p}\right)}{\check{s}\left(v_{p}, u_{p}\right)}, \\
& \check{G}_{y}=i \hat{G}_{y}=\frac{\check{p}\left(v_{p}, w_{p}\right) \check{p}\left(w_{p}, u_{p}\right)}{\check{p}\left(v_{p}, u_{p}\right)} .
\end{aligned}
$$

In general, when one describes sinusoidal waves, one has the complex notation $e^{ \pm i \omega t}$ and the real notation $(\sin \omega t, \cos \omega t)$. Similar to this, in some cases the complex notation $(\mathrm{Ai}, \mathrm{Ci})$ is convenient to describe CSR and provides a clear physical picture as in Eq. (43), but sometimes the real notation (Ai, Bi) is useful to solve a problem. We have used the complex notation to obtain the field in the frequency domain, whereas we will use the real functions for the impedance analysis and the conversion to the time domain.

\section{LONGITUDINAL IMPEDANCE}

As we stated in the Introduction, since the CSR field is proportional to the bunch charge, CSR can be described by a few geometrical parameters. We define the longitudinal impedance per unit length, $Z[\Omega / \mathrm{m}]$, as

$$
E_{s}(k)=-q_{e} \beta \lambda(k) Z(k),
$$

where the longitudinal field $E_{s}$ is evaluated at the center of the transverse beam position. By definition, the longitudinal impedance of CSR per unit length in a rectangular pipe is given by

$$
\begin{aligned}
\frac{Z(k)}{Z_{0}} & =\frac{2 \pi}{\beta h}\left(\frac{2}{k \rho}\right)^{1 / 3} \sum_{p=0}^{\infty} \Lambda_{p}\left(\hat{G}_{x}+\beta_{p}^{2} \hat{G}_{y}\right), \\
& =-\frac{2 \pi i}{\beta h}\left(\frac{2}{k \rho}\right)^{1 / 3} \sum_{p=0}^{\infty} \Lambda_{p}\left(\check{G}_{x}+\beta_{p}^{2} \check{G}_{y}\right) .
\end{aligned}
$$

$Z_{0}=c \mu_{0} \approx 120 \pi[\Omega]$ is the impedance of free space. The longitudinal impedance depends on the transverse charge distribution of the bunch. $\Lambda_{p}$ is the dimensionless form factor of the vertical charge distribution; we give some examples as follows:

$$
\Lambda_{p} \equiv \kappa h \psi_{y}^{p}= \begin{cases}1 & \text { delta function, } \\ e^{-\left(\alpha_{p} \sigma_{y}\right)^{2} / 2} & \text { Gaussian shape } \\ \frac{\sin \left(\alpha_{p} l_{y} / 2\right)}{\alpha_{p} l_{y} / 2} & \text { rectangle }\end{cases}
$$

where $\sigma_{y}$ is the rms spread of the vertical distribution if assuming Gaussian vertical distribution, $l_{y}$ is the vertical height of the bunch for the instance of a rectangular distribution. If one assumes ultrarelativistic particles: $\gamma \rightarrow$ $\infty$, as the space charge effect dies out, one can put $\Lambda_{p}=1$. If one defines the longitudinal impedance with the field value averaged over the transverse beam distribution as in [2], the form factor $\Lambda_{p}$ will be the square of Eq. (70), though it is not important in the radiation field.

The impedance of Eq. (69) is apparently imaginary, but it has singularities and contains the real impedance at the resonance points. The sharp peaks in Fig. 2 are the resonance wave numbers. The time domain field does not have a certain value until the integration contour of the Fourier transform is determined.

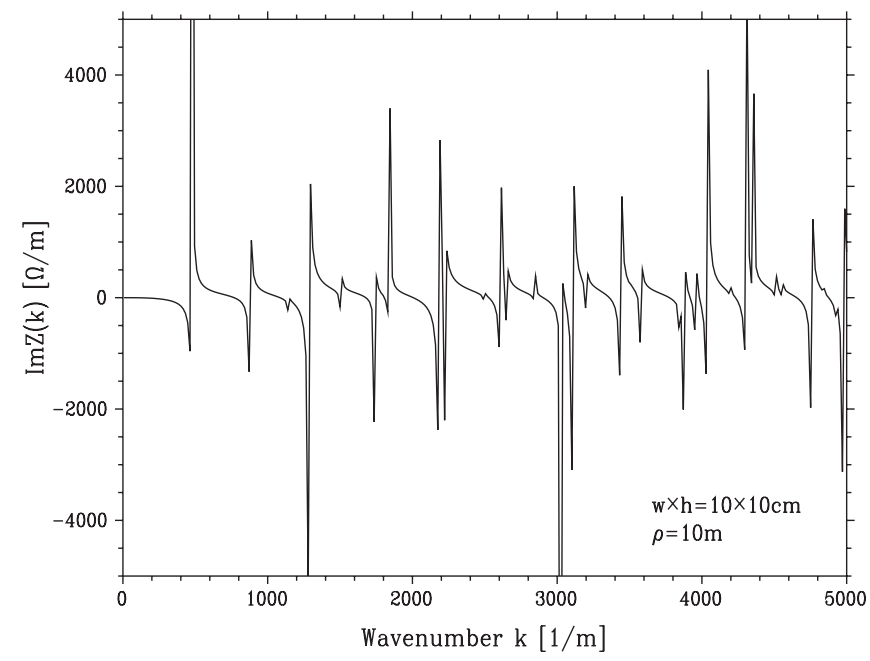

FIG. 2. CSR impedance of a square pipe. The pipe width and the height are $w \times h=10 \mathrm{~cm} \times 10 \mathrm{~cm}$, the bending radius is $\rho=10 \mathrm{~m}$. We put $\gamma=\infty$ and $\Lambda_{p}=1$ in Eq. (69). 


\section{A. Impedance of pillbox and parallel plates}

Before discussing the pole structure and the contour, let us see the impedance in a case for which the sidewalls are far away from the beam. In this limit, it must agree with the impedance of the infinite parallel plates. As we show in Sec. VI, the paraxial approximation allows us to take the limit $w \rightarrow \infty$. We will first move the inner wall away from the orbit: $x_{a} \rightarrow-\infty$ with the outer wall left at $x_{b}$. In this limit the variable $u_{p}$ of Eq. (47) goes to positive infinity; the Airy functions $\operatorname{Ai}\left(u_{p}\right)$ and $\operatorname{Ai}^{\prime}\left(u_{p}\right)$ exponentially become zero. Then we obtain the longitudinal impedance for a pillbox chamber which has a radius of $r_{b}=\rho+x_{b}$ and a height $h$,

$$
\frac{Z(k)}{Z_{0}}=\frac{2 \pi}{\beta h}\left(\frac{2}{k \rho}\right)^{1 / 3} \sum_{p=0}^{\infty} \Lambda_{p} F_{1}\left(\beta_{p}\right)
$$

where the function $F_{1}$ is given by

$$
F_{1}\left(\beta_{p}\right)=\frac{\operatorname{Ai}^{\prime}\left(w_{p}\right)}{\operatorname{Ai}^{\prime}\left(v_{p}\right)} \hat{s}\left(v_{p}, w_{p}\right)+\beta_{p}^{2} \frac{\operatorname{Ai}\left(w_{p}\right)}{\operatorname{Ai}\left(v_{p}\right)} \hat{p}\left(v_{p}, w_{p}\right) .
$$

This impedance appears imaginary but actually conceals the real part in the resonance points, i.e., at $\mathrm{Ai}^{\prime}\left(v_{p}\right)=0$ for the HE mode and at $\operatorname{Ai}\left(v_{p}\right)=0$ for the VE mode. To obtain the impedance of the pillbox chamber, it is not sufficient to move the inner wall as $x_{a} \rightarrow-\rho$ but necessary to take the limit $x_{a} \rightarrow-\infty$. Similarly, in spite of the fact that the beam pipe of Fig. 1 has infinite length with a constant curvature, rigorously speaking, our chamber model is not a torus. Appendix A accounts for this peculiar feature of our chamber model.

We will next move the outer wall away from the beam: $x_{b} \rightarrow \infty$. To do so, we must consider an energy absorption or leak on the pipe wall, otherwise the field energy is permanently preserved, the real impedance never comes up. As Warnock and Morton worked out, one can actually consider a finite conductivity on the sidewalls or the planer walls, which creates a band width on the resonances [2]. Here, instead, we use a virtual energy absorption on the wall. That is, we put an infinitesimal damping into the field: $k=k+i \epsilon$, where $\epsilon$ is a small positive parameter representing the wall resistivity. Later, this procedure will guide the integration contour of the Fourier transform to the time domain. Because of this prescription, the outgoing wave $\operatorname{Ci}\left(v_{p}\right)$ is damped as

$$
\begin{gathered}
\mathrm{Ci}\left(v_{p}\right) \simeq \frac{\left(i \epsilon \chi-v_{p}\right)^{-1 / 4}}{\sqrt{\pi}} e^{i \phi_{p}-\epsilon \chi}, \\
\phi_{p}=\frac{2}{3}\left(-v_{p}\right)^{2 / 3}-\frac{\pi}{4} \\
\chi=\frac{2 x_{b}}{3}\left(\frac{2}{k \rho}\right)^{1 / 3} \text { for } x_{b} \rightarrow \infty .
\end{gathered}
$$

$\phi_{p}$ is the phase of the outward wave; $\chi$ is a parameter of the damping whose real part is always positive for any real wave numbers including negative values for $x_{b} \rightarrow \infty$. We can now remove the outer wall: $x_{b} \rightarrow \infty$. This must be done prior to the limit $\epsilon \rightarrow+0$. Thus we obtain the impedance of the infinite parallel plates:

$$
\begin{gathered}
\frac{Z(k)}{Z_{0}}=\frac{2 \pi}{\beta h}\left(\frac{2}{k \rho}\right)^{1 / 3} \sum_{p=0}^{\infty} \Lambda_{p} F_{0}\left(\beta_{p}\right), \\
F_{0}\left(\beta_{p}\right)=\operatorname{Ai}^{\prime}\left(w_{p}\right) \mathrm{Ci}^{\prime}\left(w_{p}\right)+\beta_{p}^{2} \operatorname{Ai}\left(w_{p}\right) \operatorname{Ci}\left(w_{p}\right) .
\end{gathered}
$$

For a thin beam of ultrarelativistic particles, i.e., $\Lambda_{p}=1$ and $\gamma=\infty$, Eq. (76) agrees with Eq. (A1) of [11]. In this paper we have derived three impedances: rectangular pipe, pillbox chamber, and infinite parallel plates which are connected to Warnock-Morton's impedances $[2,4]$ via a uniform asymptotic expansion for the Bessel functions. One can obtain these impedances also from WarnockMorton's expressions as we described in Appendix A.

At last, we will remove the upper-lower plates. Let us first deal with the case for which $\gamma=\infty$; we consider a finite $\gamma$ in Sec. VI. If the upper-lower walls are infinitely far from the beam, $h \rightarrow \infty$, the sum with respect to the vertical Fourier mode in Eq. (76) becomes an integral and is exactly calculated for $\gamma=\infty$ as

$$
\begin{aligned}
& \int_{0}^{\infty} \frac{d \hat{w}}{\sqrt{\hat{w}}}\left\{\operatorname{Ai}^{\prime}(\hat{w}) \mathrm{Ci}^{\prime}(\hat{w})+\hat{w} \operatorname{Ai}(\hat{w}) \operatorname{Ci}(\hat{w})\right\} \\
& =\frac{e^{i \pi / 6}}{2 \pi}\left(\frac{2}{3}\right)^{1 / 3} \Gamma\left(\frac{2}{3}\right) .
\end{aligned}
$$

Then one obtains the well-known CSR impedance per unit length in free space [5]:

$$
\frac{Z(k)}{Z_{0}}=\frac{\Gamma(2 / 3)}{2 \pi}\left(\frac{i k}{3 \rho^{2}}\right)^{1 / 3}
$$

Equation (79) has a branch point at the origin in the complex wave number plane, which was originally located at $k= \pm \alpha_{p} / \beta$ in Warnock's impedance, given by Eq. (2.15) of [4]. They coupled at the origin due to the paraxial approximation. By Fourier transforming Eq. (79), one can calculate the time domain field as

$$
\tilde{E}_{s}(z)=-\frac{q_{e} \Gamma(2 / 3)}{(2 \pi)^{2} \varepsilon_{0}\left(3 \rho^{2}\right)^{1 / 3}} \int_{-\infty}^{\infty} \tilde{\lambda}^{\prime}\left(z^{\prime}\right) I\left(z-z^{\prime}\right) d z^{\prime},
$$

where $\tilde{\lambda}^{\prime}$ is the derivative of the line charge distribution. $I(\zeta)$ is an integral with respect to the wave number:

$$
I(\zeta)=\int_{-\infty}^{\infty} \frac{e^{i k \zeta}}{(i k)^{2 / 3}} d k
$$

We cut the complex plane along the positive (or negative) imaginary axis depending on the relation between $z$ and $z^{\prime}$ and paste three complex sheets to make a Riemann surface. 


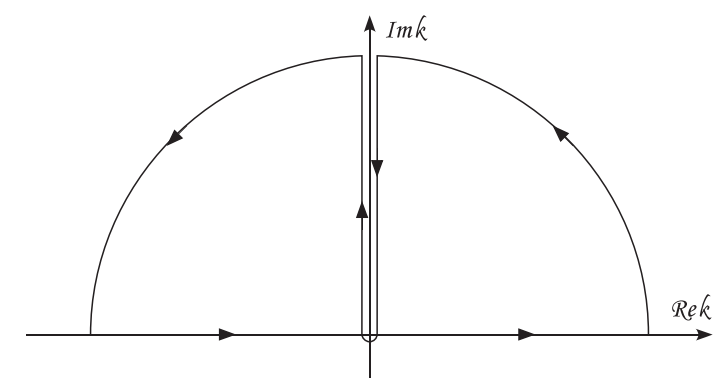

FIG. 3. Contour of free space impedance for $\zeta>0$.

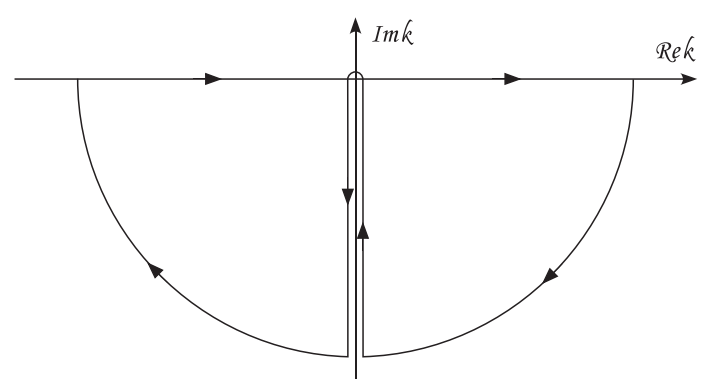

FIG. 4. Contour of free space impedance for $\zeta<0$.

Then the integral of Eq. (81) is calculated along the contours in Fig. 3 or Fig. 4.

Choosing an appropriate $\arg k$ for $\zeta>0$ and $\zeta<0$, respectively, the integral $I(\zeta)$ is calculated as

$$
I(\zeta)=\theta(\zeta) \frac{\sqrt{3}}{\zeta^{1 / 3}} \Gamma\left(\frac{1}{3}\right)
$$

where $\theta$ is the step function given by Eq. (59). Then we obtain the longitudinal field in the time domain:

$$
\tilde{E}_{s}(z)=-\frac{q_{e}}{2 \pi \varepsilon_{0}\left(3 \rho^{2}\right)^{1 / 3}} \int_{-\infty}^{z} \frac{\tilde{\lambda}^{\prime}\left(z^{\prime}\right) d z^{\prime}}{\left(z-z^{\prime}\right)^{1 / 3}} .
$$

Notice that Eq. (83) is derived from Eq. (76) without approximation. It was originally derived directly in the time domain [6,7]; on the other hand, one can derive it in the framework of the paraxial approximation. Even in the limit of $w, h \rightarrow \infty$, the paraxial approximation does not break down at high frequency: $k \rho \gg 1$. The approximation condition for the geometrical parameters will be discussed in Sec. VI in detail.

\section{B. Shielding effect in rectangular pipe}

As it is well known, the frequency components below the value given by Eq. (2) are strongly suppressed by metallic walls, therefore it is important to examine CSR impedance in the low frequency region $k<k_{\text {th }}$.

Sagan et al. investigated the shielding effect in a rectangular pipe and provided a heuristic explanation of the wall effect in Appendix A of [15]. According to their paper, the smaller one of the chamber width or the height determines the shielding effect, because transient CSR is emitted in the form of a cone which has a horizontal extent equal to the vertical one. When the cone edge hits a wall of the chamber, the CSR cone ceases to grow. Consequently, if the aspect ratio of the pipe $w / h$ is high or low, as Eq. (A1) of [15] states, the shielding effect depends on the smallest dimension. This picture is simple and intuitively clear but too rough, because Fig. 17 of [15] evidently shows that the upper-lower walls are more effective at suppressing CSR than the sidewalls.

To estimate how the sidewalls influence CSR, we will expand the CSR impedance of the rectangular pipe at low frequency. In the paraxial approximation, the longitudinal wave number must be much larger than the vertical wave number: $k \gg \alpha_{p}$ (see Appendix A), our investigation at low frequency is valid in the region $\alpha_{p} \ll k<k_{\mathrm{th}}$.

Now, we depart from the complex notation $(\mathrm{Ai}, \mathrm{Ci})$ and switch to the real one (Ai, Bi) in discussing the impedance. At first we expand the Airy functions Ai, Bi, and their derivatives using the asymptotic series for large arguments; 10.4.59-10.4.67 in [14], then we expand every part of the asymptotic expressions in Taylor series to the second order with respect to $\tau\left(=1 / 4 \beta_{p}^{3} \ll 1\right)$, where $\beta_{p}$ is the normalized vertical wave number defined in Eq. (26). We write down the intermediate calculation of the expansions in Appendix B. Here we show the asymptotic expression of the impedance in the rectangular pipe at low frequency:

$$
\frac{Z(k)}{Z_{0}}=\frac{i}{\beta h} \sum_{p=0}^{\infty} \Lambda_{p}\left[\frac{k}{\alpha_{p} \gamma^{2}} T\left(\alpha_{p} w\right)-\frac{3 k^{3}}{2 \rho^{2} \alpha_{p}^{5}} S\left(\alpha_{p} w\right)\right]
$$

where $w, h$ are the pipe width and the height, and $\alpha_{p}$ is the vertical wave number given by Eq. (25). In Eq. (84), for simplicity, we are assuming that the sidewalls are symmetric with respect to the beam: $x_{b}=-x_{a}=w / 2$. The energy is taken into consideration up to the order $1 / \gamma^{2} . S$ and $T$ are functions of the aspect ratio of the pipe:

$$
\begin{aligned}
& S(x)=\frac{\sinh x-x}{\cosh x+1}, \\
& T(x)=\frac{\sinh x}{\cosh x+1},
\end{aligned}
$$

which are shown in Fig. 5.

The first term in the bracket of Eq. (84) is the space charge field and does not depend on the curvature of the orbit. The space charge force is the Coulomb force on a straight orbit; it diverges for infinitely thin beam: $\Lambda_{p}=1$ but goes to zero for $\gamma \rightarrow \infty$. The second term in the bracket of Eq. (84) is a characteristic term on a curved orbit and 


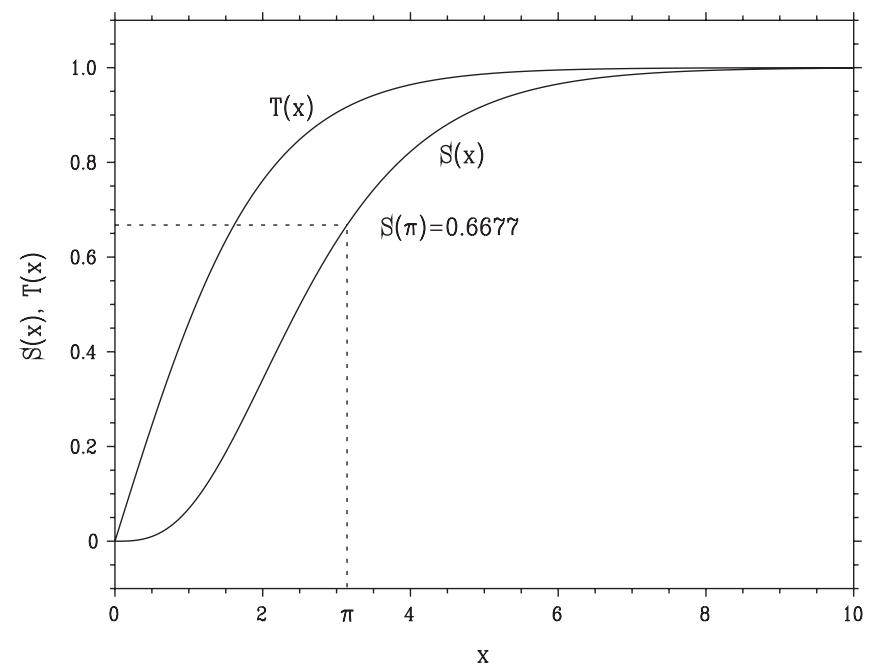

FIG. 5. Functions $S(x)$ and $T(x)$ in Eq. (84).

originates in the radiation. The radiation field does not depend on the particles energy in the high energy limit and does not diverge even for infinitely thin beam.

One may find that Eq. (84) is similar but somewhat different from Eq. (4.23) in [16]:

$$
\begin{aligned}
\frac{Z_{\mathrm{NW}}(k)}{Z_{0}}= & \frac{i}{\beta h} \sum_{p=0}^{\infty} \Lambda_{p}\left[\left(\frac{k}{\alpha_{p} \gamma^{2}}+\frac{k}{2 \rho^{2} \alpha_{p}^{3}}\right) \bar{T}\left(\alpha_{p} w\right)\right. \\
& \left.-\frac{3 k^{3}}{2 \rho^{2} \alpha_{p}^{5}} \bar{S}\left(\alpha_{p} w\right)\right],
\end{aligned}
$$

where

$$
\bar{S}(x)=1-2(x+1) e^{-x}, \quad \bar{T}(x)=1-2 e^{-x} .
$$

We are assuming again $x_{b}=-x_{a}=w / 2$, and the energy is considered to the order of $1 / \gamma^{2}$ in Eq. (87). Comparing Eq. (84) with Eq. (87), one can tell that the paraxial approximation correctly takes the space charge effect into account. It turns out, however, that Eq. (84) does not have a radiation term which is proportional to $k / \rho^{2}$, because we are assuming in the paraxial approximation that the longitudinal wave number is much larger than the vertical wave number $k \gg \alpha_{p}$; the higher order terms $\left(\alpha_{p} / k\right)^{n}(n \geq 2)$ are unfortunately neglected in our theory. For $\gamma=\infty$, the impedance of Eq. (87) is zero around $k \sim$ $\alpha_{p} / \sqrt{3}$, the impedance value is positive below it, while the value is negligibly small. Another difference is found between the functions of the aspect ratio: $S(x), T(x) \leftrightarrow$ $\bar{S}(x), \bar{T}(x)$. Considering a large aspect ratio $w / h \gg 1$, the functions $S, T$ asymptotically behave as $\bar{S}, \bar{T}$, respectively. Presumably, the difference may be caused by their somewhat rough treatment for the exponential factors in [16]. In any event, the difference between these functions yields nothing significant in the current discussion.
To examine the shielding effect by a rectangular pipe, we concentrate on the radiation term of Eq. (84) for an infinitely thin beam in the ultrarelativistic limit:

$$
\frac{Z(k)}{Z_{0}}=-i \frac{3 k^{3}}{2 h \rho^{2}} \sum_{p=0}^{\infty} \frac{S\left(\alpha_{p} w\right)}{\alpha_{p}^{5}} .
$$

In the limit that the sidewalls are far from the beam $w \rightarrow$ $\infty$, Eq. (89) agrees with the parallel plates model of Eq. (A8) of [11]. If the aspect ratio of the pipe, $w / h$, is around one or larger than one, the leading term in the sum of Eq. (89) is much larger than the sum of the rest terms and dominates the impedance value. Accordingly, we take only the leading term in the sum and neglect the higher vertical modes, then we have a simple expression:

$$
\frac{Z(k)}{Z_{0}}=-i \frac{3 k^{3}}{2 \pi \rho^{2}}\left(\frac{h}{\pi}\right)^{4} S\left(\pi \frac{w}{h}\right) .
$$

In the case of square pipe, the truncation error of the higher vertical modes is about $6.8 \times 10^{-3}$. Even if $w=h / 2$, the error stays within $2 \%$, which is not significant in the current discussion. In the low frequency region, $S(\pi w / h)$ gives the ratio of the impedances between the rectangular pipe and the parallel plates. Equation (90) is plotted with a green solid line in Fig. 6 where Ng-Warnock's equation given by Eq. (87) is also plotted with a red dashed line. At very low frequency, the discrepancy between them shows the error due to the paraxial approximation.

Equation (90) shows that, if the aspect ratio of the beam pipe is $w / h \gtrsim 1$, the impedance depends on the chamber width only through the aspect ratio in the function $S(x)$, while the upper-lower walls shield the field also by $h^{4}$ in the coefficient of the impedance. The function $S(x)$ shows the condition where one can neglect the sidewalls:

$$
w \gg \frac{h}{\pi} .
$$

In practice, as the profile of $S(x)$ shows, if the chamber width is more than 2 times larger than the height, $w>2 h$, the impedance in the low frequency hardly depends on the chamber width. Then the chamber height dominates the shielding effect, and infinite parallel plates become a good approximation for the rectangular pipe, to be shown in Fig. 12. For a shorter bunch, the low frequency components have less contribution to the field, and accordingly, the difference between the pipe and the plates will be relatively small. We obtained Eq. (91) after the asymptotic expansion of the impedance, but a simple consideration similar to that of Sagan et al. can also lead to it. That is, since CSR has a typical horizontal spread $\ell_{x}$ equal to the vertical one $\ell_{y} \sim$ $1 / \alpha_{0}=h / \pi$, comparing $\ell_{x}$ with the chamber width $w$, we immediately obtain Eq. (91). As we will see in Sec. VI A, it may be better to replace $w$ in Eq. (A1) of [15] by $(2 \pi / 3) w$. 


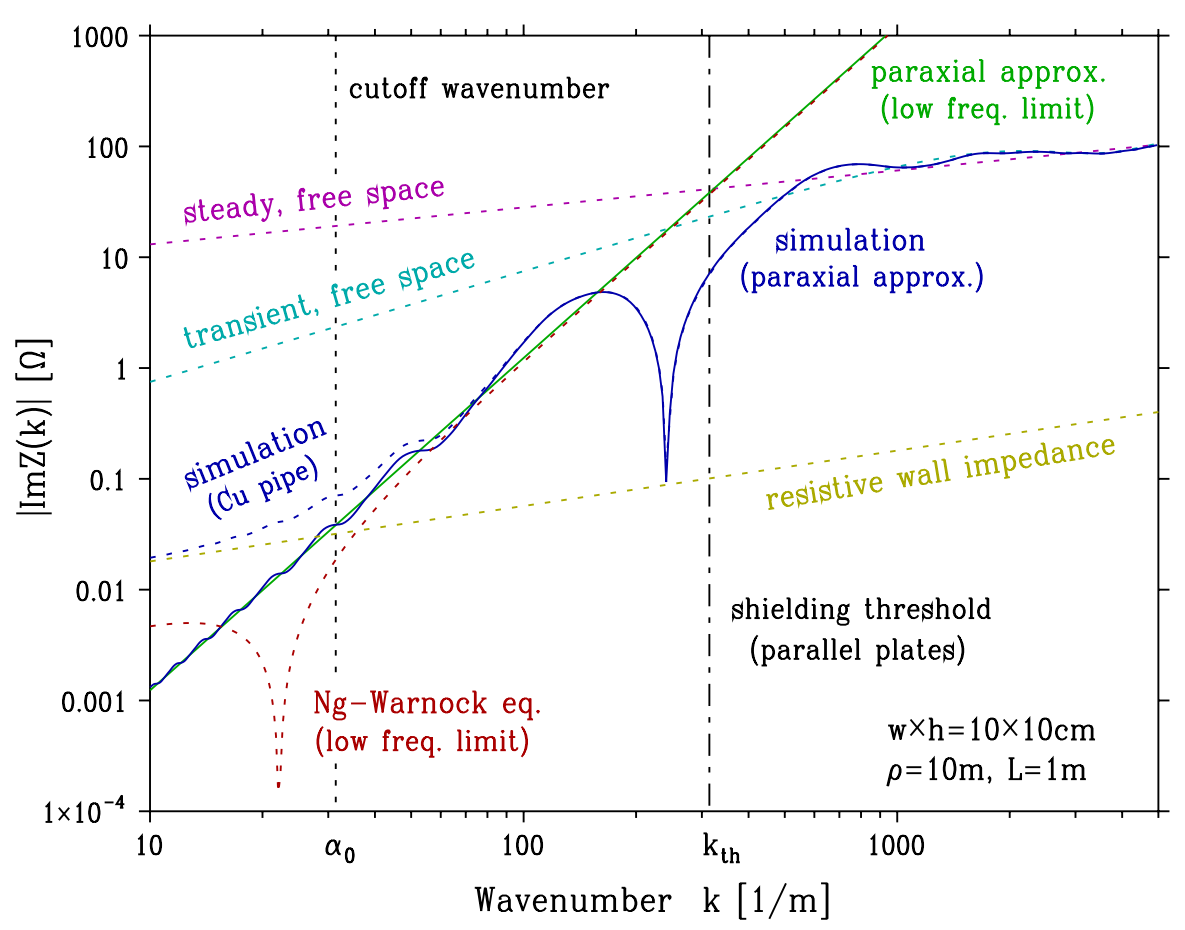

FIG. 6. (Color) Imaginary impedance in the low frequency limit. We assume a thin beam of ultrarelativistic particles, i.e., $\Lambda_{p}=1$ and $\gamma=\infty$. The green line is the asymptotic expression of Eq. (90), and the red dashed line shows Eq. (87). The blue solid line represents the simulation result using the parabolic equation in the paraxial approximation [11], where we set the magnet length to $1 \mathrm{~m}$ and assume a perfectly conducting pipe. The most realistic case is shown by the blue dashed line which is the impedance in a copper square pipe, obtained by simulation [12]. The chamber width and the height are $w \times h=10 \mathrm{~cm} \times 10 \mathrm{~cm}$, and the bending radius is $\rho=$ $10 \mathrm{~m}$. The dashed vertical line is the vertical cutoff wave number $\alpha_{0}=\pi / h$, and the dot-dashed vertical line is the threshold wave number $k_{\mathrm{th}}$. We draw also the CSR impedance in free space: the steady CSR of Eq. (79) is shown by the magenta dashed line and the transient state [8] by the cyan dashed line. The brown dashed line is the resistive wall impedance [18] due to a copper round pipe which is straight and has a diameter of $10 \mathrm{~cm}$. (It is shown that the longitudinal resistive wall wakefield at the center of a square pipe is equal to the one at the center of a round pipe which is inscribed in the square $[12,19]$.)

\section{FIELD RESONANCE}

If the radiation emitted from particles has a phase velocity equal to the particle velocity in a curved pipe, the radiation synchronously interacts with the particles and can propagate in the pipe. This is the resonance of the radiation field in a curved pipe, which cannot occur between superimposed parallel plates, because the radiation goes away off the beam.

\section{A. Resonance poles}

In a rectangular pipe, the resonance points of the HE/VE mode are determined, respectively, by solving

$$
\begin{array}{cl}
\breve{s}\left[v_{p}(k), u_{p}(k)\right]=0 & \text { HE mode, } \\
\check{p}\left[v_{p}(k), u_{p}(k)\right]=0 & \text { VE mode, }
\end{array}
$$

where $u_{p}, v_{p}$ are functions of the wave number, and we rewrite their definition:

$$
u_{p}(k)=\frac{\alpha_{p}^{2}}{\kappa^{2}}-\kappa \rho\left(\frac{x_{a}}{\rho}-\frac{1}{2 \gamma^{2}}\right),
$$

$$
v_{p}(k)=\frac{\alpha_{p}^{2}}{\kappa^{2}}-\kappa \rho\left(\frac{x_{b}}{\rho}-\frac{1}{2 \gamma^{2}}\right) .
$$

They are variables which are concerned with the inner wall position and the outer wall position, respectively. As we mentioned in Sec. II B, the energy deviation of the particles is related to the horizontal displacement,

$$
\delta x=\frac{\rho}{2 \gamma^{2}} .
$$

The ultrarelativistic limit is available if

$$
\frac{1}{\gamma} \ll \min \left[\frac{\pi \sigma_{z}}{h}, \sqrt{\frac{w}{\rho}}\right] .
$$

In contrast, low energy particles such as

$$
\frac{1}{\gamma}>\sqrt{\frac{2 x_{b}}{\rho}}
$$

cannot radiate constantly in the beam pipe except in a transient state around the entrance of the magnet. Equa- 
tion (97) is an approximate condition which corresponds to Eq. (3) of [2] and Eq. (1) of [3].

In the following of this subsection, we put $\gamma=\infty$ for simplicity, which does not spoil the argument of the field resonance. If a finite energy is to be considered, one can simply replace the sidewall positions $x_{i}$ by $x_{i}-\delta x(i=a$, $b)$. Several authors obtained the approximate resonance points in a toroidal chamber at high frequency $[2,3,10]$. We write the resonance points using our notation:

$$
k_{m}^{p}=\alpha_{p} \sqrt{\frac{\rho}{x_{b}}} \Upsilon\left(\frac{h(m-d)}{x_{b}(2 p+1)}\right) \quad\left(\begin{array}{c}
m=1,2,3,4, \ldots \\
p=0,1,2,3, \ldots
\end{array}\right),
$$

where $d$ is the following constant:

$$
d= \begin{cases}3 / 4 & \text { HE mode } \\ 1 / 4 & \text { VE mode }\end{cases}
$$

$\Upsilon(r)$ is a monotonically increasing function:

$$
Y(r)=\left[\left(\sqrt{1+\frac{r^{2}}{3}}+1\right)^{1 / 3}-\left(\sqrt{1+\frac{r^{2}}{3}}-1\right)^{1 / 3}\right]^{-3 / 2}
$$

which behaves as $Y(r) \sim 3 r / 2^{3 / 2}$ for $r$ large. The solution is given in Sec. VI. In Eq. (98), $m$ is the resonance mode index, and $p$ is the mode index of the vertical Fourier series. As Eq. (98) shows, the HE/VE resonances appear alternately on the wave number axis. In Eq. (98) it may be better to put $m-3 / 4=n+1 / 4(n=0,1,2, \cdots)$ only for the HE mode, because then the indices $n, m$ correspond to the number of nodes, respectively, for the HE/VE mode in the radial dimension of the pipe [10]. However, for convenience in describing the time domain field in Sec. V, we use the index $m(=1,2,3, \ldots)$ both for the $\mathrm{HE}$ and the VE mode. Equation (98) also implies that the resonance wave numbers at high frequency do not depend on the inner wall position $x_{a}$ but is determined by the outer wall position $x_{b}$ and the chamber height $h$. This means that the field in a toroidal chamber is similar to the one in a pillbox chamber unless the inner wall of the pipe is very close to the beam [2]. Actually, Eq. (98) was derived from the resonance condition of the pillbox chamber [see Eqs. (72) and (182)].

The accuracy of Eq. (98) is discussed in [10]. The maximum error occurs at the lowest resonance: $m=1$. The error is just about 5\% which is not so bad; however, since the field rapidly depends on the lower resonance points as to be shown in Figs. 9 and 10, the approximate solution of Eq. (98) can yield a large error in the field. For a square pipe, the error by using the approximate poles is about $30 \%$ in the field value; the error becomes small as the pipe width goes to infinity. To obtain the field value with a good accuracy, we have to rely on a numerical calculation. As one will see in Sec. VI, if the lowest resonance mode is much smaller than the shielding threshold, i.e., $k_{1} \ll k_{\text {th }}$, the field does not depend on the resonance points. Equation (98) is important in understanding the pole structure of the radiation field in a curved rectangular pipe. In addition, as it is remarked in [2], the approximate poles can be utilized as initial values of a Newton search for solving Eqs. (92).

\section{B. Imaginary poles}

Now, we will go to our main business. We suppose that the wave number is purely imaginary:

$$
k=i \bar{k}
$$

where $\bar{k} \in \mathbb{R}$ is assumed to have a positive value for the moment, but it can be negative in general. For imaginary wave numbers, the function $\kappa=\left(2 k^{2} / \rho\right)^{1 / 3}$ becomes

$$
\kappa(i \bar{k})=c \bar{\kappa},
$$

where $c$ is a complex constant,

$$
c \equiv(-1)^{1 / 3}=\left\{-1, e^{ \pm i \pi / 3}\right\} .
$$

$\bar{\kappa}$ is similar to $\kappa$ but defined only for real wave numbers hence always real:

$$
\bar{\kappa}=\kappa(\bar{k}) .
$$

For imaginary wave numbers, the arguments of the Airy functions, $u_{p}$ and $v_{p}$, are given as follows:

$$
\begin{aligned}
& u_{p}(i \bar{k})=c^{-2}\left[\frac{\alpha_{p}^{2}}{\bar{\kappa}^{2}}+\bar{\kappa} \rho\left(\frac{x_{a}}{\rho}-\frac{1}{2 \gamma^{2}}\right)\right], \\
& v_{p}(i \bar{k})=c^{-2}\left[\frac{\alpha_{p}^{2}}{\bar{\kappa}^{2}}+\bar{\kappa} \rho\left(\frac{x_{b}}{\rho}-\frac{1}{2 \gamma^{2}}\right)\right] .
\end{aligned}
$$

We give a simple example to illustrate the pole structure of the impedance. At first, we put $\gamma=\infty$. Suppose that the beam pipe has horizontal symmetry: $x_{a}=-x_{b}$, then for a imaginary wave number, the variables of the Airy functions are given by

$$
\begin{aligned}
& u_{p}(i \bar{k})=c^{-2} v_{p}(\bar{k}), \\
& v_{p}(i \bar{k})=c^{-2} u_{p}(\bar{k}) .
\end{aligned}
$$

If we choose $c=-1$ in Eq. (103), we have $u_{p}(i \bar{k})=v_{p}(\bar{k})$ and $v_{p}(i \bar{k})=u_{p}(\bar{k})$; that is, the role of the outer wall is exactly replaced by the inner wall. This means that there exist imaginary wave numbers which satisfy the resonance conditions given by Eqs. (92). If $\gamma=\infty$ and $x_{a}=-x_{b}$, the imaginary poles are symmetric to the real ones and the residues at the poles have a symmetry between the real axis and the imaginary axis. Although we have shown above a special case, even if the sidewalls are not symmetric with respect to the beam, the cross products $\breve{s}\left(v_{p}, u_{p}\right)$ and $\check{p}\left(v_{p}, u_{p}\right)$ have zeros not only on the real axis but also on the imaginary axis. If the sidewalls are not symmetric or if 
the Lorentz factor is finite, the poles on the imaginary axis are not symmetric with respect to the real axis. They are gotten by solving

$$
\begin{array}{ll}
\check{s}\left[\bar{v}_{p}(\bar{k}), \bar{u}_{p}(\bar{k})\right]=0 & \text { HE mode, } \\
\check{p}\left[\bar{v}_{p}(\bar{k}), \bar{u}_{p}(\bar{k})\right]=0 & \text { VE mode, }
\end{array}
$$

where we defined $\bar{u}_{p}(k), \bar{v}_{p}(k)$, and $\bar{w}_{p}(k)$ for $\forall k \in \mathbb{C}$ as

$$
\begin{gathered}
\bar{u}_{p}(k)=\frac{\alpha_{p}^{2}}{\kappa^{2}}+\kappa \rho\left(\frac{x_{a}}{\rho}-\frac{1}{2 \gamma^{2}}\right), \\
\bar{v}_{p}(k)=\frac{\alpha_{p}^{2}}{\kappa^{2}}+\kappa \rho\left(\frac{x_{b}}{\rho}-\frac{1}{2 \gamma^{2}}\right), \\
\bar{w}_{p}(k)=\frac{\alpha_{p}^{2}}{\kappa^{2}}-\frac{\kappa \rho}{2 \gamma^{2}} .
\end{gathered}
$$

The variables of the Airy functions $u_{p}, v_{p}$, and $w_{p}$ have the following property in the complex $k$ plane:

$$
\begin{gathered}
u_{p}\left(k^{*}\right)=u_{p}^{*}(k), \\
v_{p}\left(k^{*}\right)=v_{p}^{*}(k), \\
w_{p}\left(k^{*}\right)=w_{p}^{*}(k) .
\end{gathered}
$$

The asterisk denotes the complex conjugate. In addition, the Airy functions have mirror symmetry for $\forall z \in \mathbb{C}$ [17],

$$
\mathrm{Xi}\left(z^{*}\right)=\mathrm{Xi}^{*}(z),
$$

where $\mathrm{Xi}$ represents either $\mathrm{Ai}, \mathrm{Bi}, \mathrm{Ai}^{\prime}$, or $\mathrm{Bi}^{\prime}$. For a purely imaginary wave number which satisfies the resonance condition of Eq. (92a) or (92b), the complex conjugate satisfies the same condition:

$$
\begin{array}{ll}
\breve{s}\left[v_{p}\left(k^{*}\right), u_{p}\left(k^{*}\right)\right]=0 & \text { HE mode, } \\
\check{p}\left[v_{p}\left(k^{*}\right), u_{p}\left(k^{*}\right)\right]=0 & \text { VE mode. }
\end{array}
$$

Namely, the negative imaginary axis has a pole structure symmetric to the positive imaginary axis. If one chooses another value: $c=e^{ \pm i \pi / 3}$ in Eq. (103), the resonance points on the imaginary axis are the same as for $c=-1$ (Appendix C). In addition, the impedance does not depend on the value choice of $c$ and obeys the rule

$$
Z(-k)=Z^{*}(k) .
$$

If $x_{a}=-x_{b}$ and $\gamma=\infty$, it has the following symmetry:

$$
Z( \pm i k)=\mp i Z(k) \text {. }
$$

Let us obtain the approximate poles on the imaginary axis for large $|k|$. As $\bar{v}_{p}(\bar{k})$ is positive large for $\bar{k}$ large, the Airy function $\operatorname{Ai}\left(\bar{v}_{p}\right)$ and $\operatorname{Ai}^{\prime}\left(\bar{v}_{p}\right)$ become negligible. Accordingly, the cross products $\breve{p}\left(\bar{v}_{p}, \bar{u}_{p}\right), \check{s}\left(\bar{v}_{p}, \bar{u}_{p}\right)$ behave for large $\bar{k}$ as

$$
\begin{aligned}
\check{s}\left(\bar{v}_{p}, \bar{u}_{p}\right) \simeq-\mathrm{Bi}^{\prime}\left(\bar{v}_{p}\right) \mathrm{Ai}^{\prime}\left(\bar{u}_{p}\right), \\
\check{p}\left(\bar{v}_{p}, \bar{u}_{p}\right) \simeq-\operatorname{Bi}\left(\bar{v}_{p}\right) \operatorname{Ai}\left(\bar{u}_{p}\right) .
\end{aligned}
$$

$\operatorname{Bi}\left(\bar{v}_{p}\right)$ and $\operatorname{Bi}^{\prime}\left(\bar{v}_{p}\right)$ are exponential functions and have no zeros for any wave number on the imaginary axis. Therefore the approximate imaginary poles are obtained by solving

$$
\begin{aligned}
\operatorname{Ai}^{\prime}\left(\bar{u}_{p}\right)=0 & \text { HE mode } \\
\operatorname{Ai}\left(\bar{u}_{p}\right)=0 & \text { VE mode }
\end{aligned}
$$

Recall that the variable $\bar{u}_{p}$ is defined by Eq. (110) and depends on the inner wall position $x_{a}$. The imaginary poles are determined by the inner wall position and the chamber height; they do not depend on the outer wall position for $|k|$ large. But this fact seems strange, because CSR has a typical horizontal expanse $\ell_{x} \sim h / \pi$ in a pipe satisfying $w>h / \pi$, so it is hard to understand that the inner wall is involved to determine the field, unless the beam travels very close to the inner wall.

For negative large $\bar{u}_{p}$, the Airy functions $\operatorname{Ai}\left(\bar{u}_{p}\right)$ and $\operatorname{Ai}^{\prime}\left(\bar{u}_{p}\right)$ asymptotically behave as

$$
\begin{aligned}
\operatorname{Ai}^{\prime}\left(\bar{u}_{p}\right) \simeq-\frac{\left(-\bar{u}_{p}\right)^{1 / 4}}{\sqrt{\pi}} \cos \left(\zeta_{\bar{u}}+\frac{\pi}{4}\right), \\
\operatorname{Ai}\left(\bar{u}_{p}\right) \simeq \frac{\left(-\bar{u}_{p}\right)^{-1 / 4}}{\sqrt{\pi}} \sin \left(\zeta_{\bar{u}}+\frac{\pi}{4}\right), \\
\zeta_{\bar{u}}=(2 / 3)\left(-\bar{u}_{p}\right)^{3 / 2} \in \mathbb{R},
\end{aligned}
$$

and the zeros of $\operatorname{Ai}\left(\bar{u}_{p}\right), \operatorname{Ai}^{\prime}\left(\bar{u}_{p}\right)$ are approximately given by

$$
\zeta_{\bar{u}}=\pi(m-d)
$$

$d$ is the constant given by Eq. (99). We define a variable $X$ and coefficient $a$ as

$$
\begin{gathered}
X=\frac{1}{\bar{\kappa}}\left(\frac{\alpha_{p}^{2}}{-x_{a}}\right)^{1 / 3}, \\
a=\left\{\frac{3 \zeta_{\bar{u}}}{2 \alpha_{p}\left(-x_{a}\right)}\right\}^{2 / 3} .
\end{gathered}
$$

Equations (124) and (125) give a cubic equation to be solved:

$$
X^{3}+a X-1=0 .
$$

Cardano's formula for the cubic equation gives the approximate poles on the positive imaginary axis: $k=i \bar{k}_{m}^{p}$,

$$
\bar{k}_{m}^{p}=\alpha_{p} \sqrt{\frac{\rho}{-x_{a}}} \Upsilon\left(\frac{h(m-d)}{x_{a}(2 p+1)}\right) \quad\left(\begin{array}{c}
m=1,2,3,4, \ldots \\
p=0,1,2,3, \ldots
\end{array}\right) .
$$

$\Upsilon(r)$ is a function given by Eq. (100). Because of the mirror 
symmetry of the Airy functions, the poles on the negative imaginary axis are symmetric to the ones on the positive imaginary axis: $i \bar{k}_{-m}^{p}=-i \bar{k}_{m}^{p}$. If the sidewalls are symmetric, $x_{a}+x_{b}=0$, and if the particles are ultrarelativistic, the approximate poles are symmetric between the real axis and the imaginary axis, $\bar{k}_{m}^{p}=k_{m}^{p}$. The above consideration has shown that the inner wall of the pipe mainly determines the imaginary poles; on the other hand, the outer wall by and large determines the real resonance poles.

\section{FIELDS IN THE TIME DOMAIN}

\section{A. Fourier transform and contour}

In deriving the time domain field, we assume ultrarelativistic particles. Since the causality of the field is complicated for a finite $\gamma$, we hope to discuss it elsewhere in the future. In this section we consider a finite $\gamma$ only for the expression of the impedance. The longitudinal field in the time domain is given by

$$
\tilde{E}_{s}(z)=-\frac{q_{e}}{2 \pi} \int_{-\infty}^{\infty} \beta \lambda(k) Z(k) e^{i k z} d k .
$$

We use the real notation (Ai, Bi) for the impedance in this section. In advance, we rewrite the bunch spectrum of Eq. (9) using integration by parts,

$$
\lambda(k)=\int_{-\infty}^{\infty} d z^{\prime} \frac{e^{-i k z^{\prime}}}{i k} \frac{d \tilde{\lambda}\left(z^{\prime}\right)}{d z^{\prime}} .
$$

Substitution of Eq. (131) turns the integral with respect to the wave number into a $z$ integral,

$$
\tilde{E}_{s}(z)=\frac{q_{e}}{\varepsilon_{0} h} \int_{-\infty}^{\infty} d z^{\prime} \frac{d \tilde{\lambda}\left(z^{\prime}\right)}{d z^{\prime}} \sum_{p=0}^{\infty} \Lambda_{p} I_{p}\left(z-z^{\prime}\right),
$$

where $\varepsilon_{0}$ is the permittivity of vacuum. $I_{p}$ is the integral with respect to the longitudinal wave number:

$$
I_{p}(\zeta)=\int_{-\infty}^{\infty} \frac{\kappa d k}{k^{2}}\left(\check{G}_{x}+\beta_{p}^{2} \check{G}_{y}\right) e^{i k \zeta},
$$

which converges for $k \in \mathbb{R} \rightarrow \pm \infty$ owing to the integration by parts of Eq. (131), because the impedance behaves as $Z(k) \propto(i k)^{1 / 3}$ in the high frequency limit for $\gamma=\infty$. The variable $\zeta$ of Eq. (133) is the longitudinal distance between the observation point $z$ and the position of the field particle $z^{\prime}$ :

$$
\zeta=z-z^{\prime}
$$

The integral $I_{p}$ requires a case separation depending on the relation between the observer and the field particle so that the field is bounded:

$$
\begin{array}{ll}
\Im k>0 & \text { for } \zeta>0 \text { : ahead of source, } \\
\Im k<0 & \text { for } \zeta<0 \text { : behind source. }
\end{array}
$$

That is, when the observation point is ahead of the field source, we must close the contour in the upper half plane, otherwise the integral $I_{p}$ diverges, and vise versa. If one considers a finite $\gamma$ in $I_{p}$, the case separation of Eq. (135) is, precisely speaking, incorrect, because $\check{G}_{x}+\beta_{p}^{2} \breve{G}_{y}$ in $I_{p}$ could asymptotically behave as an exponential function for $w, h \rightarrow \infty$, and $\Im k \rightarrow \pm \infty$. The integral of Eq. (208) suggests that the critical wave number $k_{c}\left(=3 \gamma^{3} / 2 \rho\right)$ may be involved in the field causality. We do not have the asymptotic expression of the impedance with a finite $\gamma$ in the limit $w, h \rightarrow \infty$, and $\Im k \rightarrow \pm \infty$. Therefore we put $\gamma=\infty$ in deriving the time domain field.

To determine the contour on the real axis, we must consider an infinitesimal damping for the outward waves,

$$
k=\lim _{\epsilon \rightarrow+0}(k+i \epsilon)
$$

or the field does not contain a real impedance as it is shown in Sec. III A. In Eq. (136), $\epsilon$ is a small positive parameter representing the infinitesimal energy loss on the pipe. Therefore, the contour on the real line must go through over the real poles. In addition, by Taylor series expansion around each pole, it is readily shown that all real and imaginary poles are simple. As we have shown in Sec. III B, the impedance has no pole nor branch point at the origin, the integrand of $I_{p}$ is also regular at the origin. Therefore the contours of Figs. 7 and 8 satisfy the physical requirements of our problem.

These contours determine the impedance value which has simple poles on the real axis, i.e.,

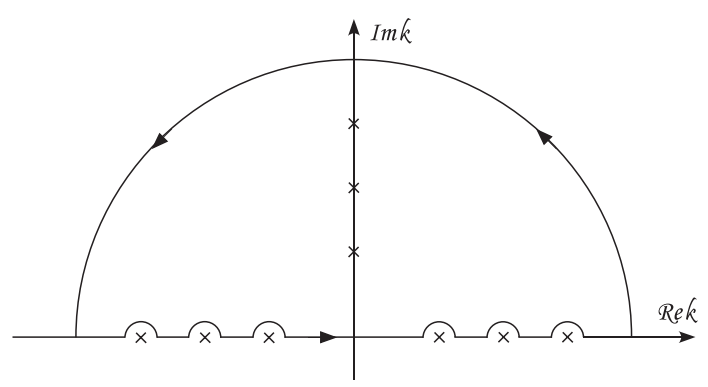

FIG. 7. Contour for $\zeta>0$.

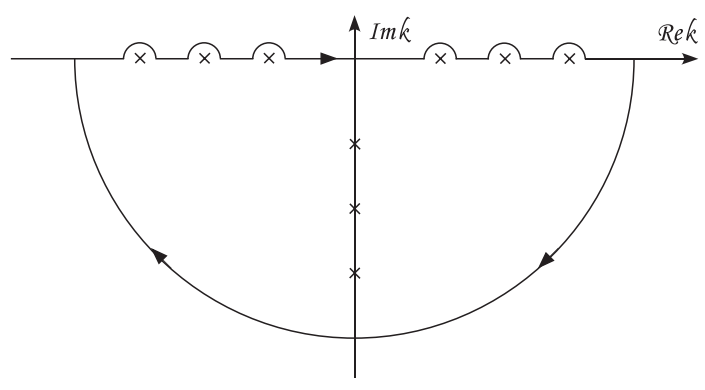

FIG. 8. Contour for $\zeta<0$. 


$$
\frac{1}{k-k_{m}+i \epsilon}=\mathcal{P} \frac{1}{k-k_{m}}-i \pi \delta\left(k-k_{m}\right)
$$

where the symbol $\mathcal{P}$ indicates the principal value of the integral. The wave number $k_{m}$ represents a real resonance point of either the HE mode or the VE mode,

$$
k_{m}= \begin{cases}\mu_{m}^{p}(\mathrm{HE}) ; & \check{s}\left[v_{p}\left(\mu_{m}^{p}\right), u_{p}\left(\mu_{m}^{p}\right)\right]=0, \\ \nu_{m}^{p}(\mathrm{VE}) ; & \check{p}\left[v_{p}\left(\nu_{m}^{p}\right), u_{p}\left(\nu_{m}^{p}\right)\right]=0 .\end{cases}
$$

The imaginary part of the impedance is the principal value of Eq. (69): $\Im Z(k)=\mathcal{P} Z(k)$. On the other hand, the second term on the right-hand side of Eq. (137) corresponds to the real impedance:

$$
\begin{gathered}
\frac{\Re Z(k)}{Z_{0}}=-\frac{2 \pi^{2}}{\beta h} \sum_{p=0}^{\infty} \Lambda_{p} \sum_{\substack{m=-\infty \\
m \neq 0}}^{\infty} D_{m}^{p}(k), \\
D_{m}^{p}(k)=R_{x}^{p}(k) \delta\left(k-\mu_{m}^{p}\right)+R_{y}^{p}(k) \delta\left(k-\nu_{m}^{p}\right),
\end{gathered}
$$

where we consider a finite $\gamma$. The total energy loss of the bunch per unit length is obtained from Eq. (139):

$$
\begin{gathered}
\frac{d \mathcal{E}_{\mathrm{b}}}{d s}=\frac{2 \pi q_{e}^{2}}{\varepsilon_{0} h} \sum_{p=0}^{\infty} \Lambda_{p} \sum_{m=1}^{\infty} L_{m}^{p}, \\
L_{m}^{p}=R_{x}^{p}\left(\mu_{m}^{p}\right)\left|\lambda\left(\mu_{m}^{p}\right)\right|^{2}+R_{y}^{p}\left(\nu_{m}^{p}\right)\left|\lambda\left(\nu_{m}^{p}\right)\right|^{2} .
\end{gathered}
$$

Omitting the coefficients, $R_{x}^{p}\left(\mu_{m}^{p}\right)$ and $R_{y}^{p}\left(\nu_{m}^{p}\right)$ are the residues of the impedance at the real poles of the HE/VE mode, respectively. As a function of the wave number, they are given by

$$
\begin{aligned}
& R_{x}^{p}(k)=\frac{\kappa}{k} \frac{\check{s}\left(v_{p}, w_{p}\right) \check{s}\left(w_{p}, u_{p}\right)}{\check{s}^{\prime}\left(v_{p}, u_{p}\right)}, \\
& R_{y}^{p}(k)=\beta_{p}^{2} \frac{\kappa}{k} \frac{\check{p}\left(v_{p}, w_{p}\right) \check{p}\left(w_{p}, u_{p}\right)}{\check{p}^{\prime}\left(v_{p}, u_{p}\right)},
\end{aligned}
$$

where the primes in the denominators denote the derivative with respect to the wave number $k$,

$$
\begin{aligned}
& \frac{d \check{s}\left(v_{p}, u_{p}\right)}{d k}=\frac{2 \kappa}{3 k}\left(u_{p} \frac{d u_{p}}{d \kappa} \breve{r}\left(v_{p}, u_{p}\right)+v_{p} \frac{d v_{p}}{d \kappa} \breve{q}\left(v_{p}, u_{p}\right)\right), \\
& \frac{d \check{p}\left(v_{p}, u_{p}\right)}{d k}=\frac{2 \kappa}{3 k}\left(\frac{d u_{p}}{d \kappa} \breve{q}\left(v_{p}, u_{p}\right)+\frac{d v_{p}}{d \kappa} \check{r}\left(v_{p}, u_{p}\right)\right) .
\end{aligned}
$$

The cross products $\breve{p}, \check{q}, \breve{r}$, and $\breve{s}$ are given by Eqs. (63). $R_{x}^{p}(k)$ and $R_{y}^{p}(k)$ are functions having dimension of inverse length, which are shown in Figs. 9 and 10 with the resonance points of the HE/VE mode. As these figures show,

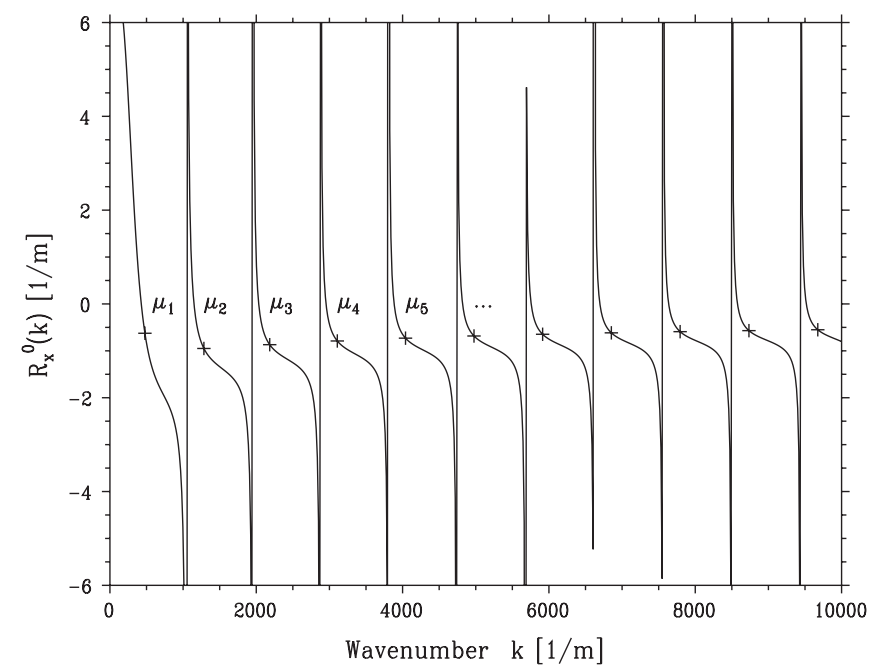

FIG. 9. Function $R_{x}^{p}(k)$ and the resonance points $\mu_{m}^{p}$ for the fundamental vertical mode $(p=0)$. The symbols $(+)$ on the line are the resonance points of the HE mode which are obtained numerically by a Newton search. The parameters used here are as follows: $x_{b}=-x_{a}=5 \mathrm{~cm}, h=10 \mathrm{~cm}, \rho=10 \mathrm{~m}$, and $\gamma=\infty$

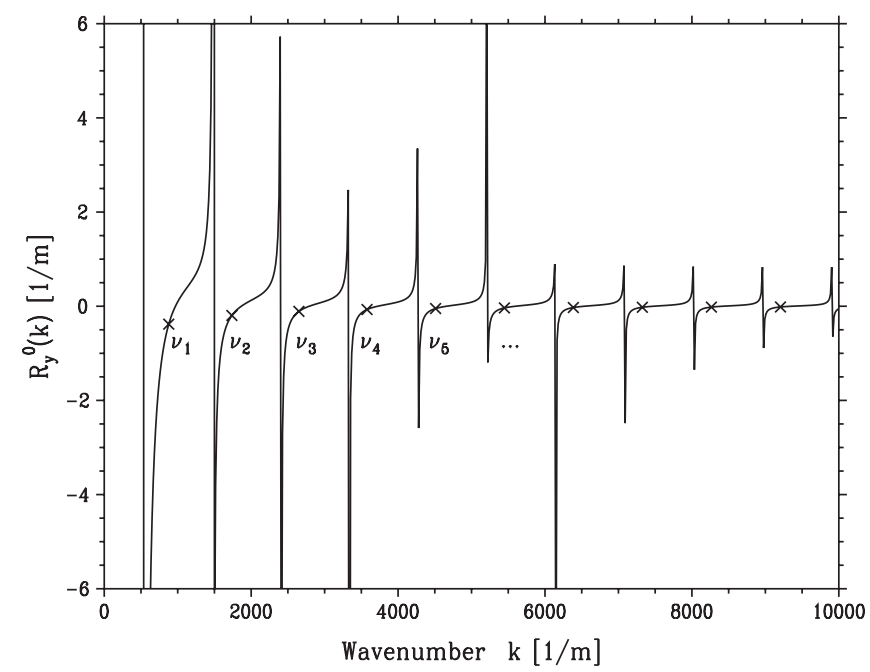

FIG. 10. Function $R_{y}^{p}(k)$ and the resonance points $\nu_{m}^{p}$ for $p=$ 0 . The symbols $(X)$ are the resonance points of the VE mode. The parameters are the same as Fig. 9.

since $R_{x}^{p}, R_{y}^{p}$ can rapidly change, a small error in the lower resonance points can yield a large error to the field value. Using the following cyclic identities,

$$
\begin{aligned}
& \operatorname{Bi}^{\prime}\left(w_{p}\right) \check{s}\left(v_{p}, u_{p}\right)=\operatorname{Bi}^{\prime}\left(u_{p}\right) \check{s}\left(v_{p}, w_{p}\right)+\operatorname{Bi}^{\prime}\left(v_{p}\right) \check{s}\left(w_{p}, u_{p}\right), \\
& \operatorname{Bi}\left(w_{p}\right) \check{p}\left(v_{p}, u_{p}\right)=\operatorname{Bi}\left(u_{p}\right) \check{p}\left(v_{p}, w_{p}\right)+\operatorname{Bi}\left(v_{p}\right) \check{p}\left(w_{p}, u_{p}\right),
\end{aligned}
$$

with Eq. (138), we obtain $R_{x}^{p}$ and $R_{y}^{p}$ at the resonance 
points of the HE/VE mode:

$$
\begin{aligned}
& R_{x}^{p}\left(\mu_{m}^{p}\right)=-\frac{3 \pi}{2} \frac{\check{s}^{2}\left(v_{p}, w_{p}\right)}{v_{p} \frac{d v_{p}}{d \kappa}-u_{p} \frac{d u_{p}}{d \kappa}\left\{\frac{\mathrm{Bi}^{\prime}\left(v_{p}\right)}{\mathrm{Bi}^{\prime}\left(u_{p}\right)}\right\}^{2}}, \\
& R_{y}^{p}\left(\nu_{m}^{p}\right)=+\frac{3 \pi}{2} \frac{\beta_{p}^{2} \breve{p}^{2}\left(v_{p}, w_{p}\right)}{\frac{d v_{p}}{d \kappa}-\frac{d u_{p}}{d \kappa}\left\{\frac{\operatorname{Bi}\left(v_{p}\right)}{\operatorname{Bi}\left(u_{p}\right)}\right\}^{2}},
\end{aligned}
$$

where the functions of the wave number, $u_{p}, v_{p}, w_{p}, \beta_{p}$, and $\kappa$ on the right-hand sides, are evaluated at the HE and the VE resonances, respectively. Ignoring the coefficients, Eqs. (146) correspond to Eqs. (114) and (115) of [2]. The second terms in the denominators of Eqs. (146) are smaller than the first terms, therefore both $R_{x}^{p}\left(\mu_{m}^{p}\right)$ and $R_{y}^{p}\left(\nu_{m}^{p}\right)$ are always negative for any $m$ and $p$.

\section{B. Time domain field}

The contour of the Fourier transform is not unique; one can choose another pair of contours for the integral $I_{p}$ as is possible for CSR impedance in free space, $Z \propto(i k)^{1 / 3}$, shown in Figs. 3 and 4 where the real poles must be infinitesimally lowered off the real axis. According to the contours shown in Figs. 7 and 8, or Figs. 3 and 4, the integral $I_{p}$ is calculated as

$$
\begin{aligned}
\frac{I_{p}(\zeta)}{2 \pi i}= & \theta(\zeta) \sum_{m=1}^{\infty}\left(\frac{R_{x}^{p}\left(i \bar{\mu}_{m}^{p}\right)}{i \bar{\mu}_{m}^{p}} e^{-\bar{\mu}_{m}^{p} \zeta}+\frac{R_{y}^{p}\left(i \bar{\nu}_{m}^{p}\right)}{i \bar{\nu}_{m}^{p}} e^{-\bar{\nu}_{m}^{p} \zeta}\right) \\
& -\theta(-\zeta) \sum_{m=-\infty}^{-1}\left(\frac{R_{x}^{p}\left(i \bar{\mu}_{m}^{p}\right)}{i \bar{\mu}_{m}^{p}} e^{-\bar{\mu}_{m}^{p} \zeta}+\frac{R_{y}^{p}\left(i \bar{\nu}_{m}^{p}\right)}{i \bar{\nu}_{m}^{p}} e^{-\bar{\nu}_{m}^{p} \zeta}\right) \\
& -\theta(-\zeta) \sum_{\substack{m=-\infty \\
m \neq 0}}^{\infty}\left(\frac{R_{x}^{p}\left(\mu_{m}^{p}\right)}{\mu_{m}^{p}} e^{i \mu_{m}^{p} \zeta}+\frac{R_{y}^{p}\left(\nu_{m}^{p}\right)}{\nu_{m}^{p}} e^{i \nu_{m}^{p} \zeta}\right),
\end{aligned}
$$

where $\theta$ is the step function. $\bar{\mu}_{m}^{p}, \bar{\nu}_{m}^{p} \in \mathbb{R}$ are the poles on the imaginary axis for the HE/VE mode, respectively,

$$
\bar{k}_{m}= \begin{cases}\bar{\mu}_{m}^{p}(\mathrm{HE}) ; & \breve{s}\left[\bar{v}_{p}\left(\bar{\mu}_{m}^{p}\right), \bar{u}_{p}\left(\bar{\mu}_{m}^{p}\right)\right]=0, \\ \bar{\nu}_{m}^{p}(\mathrm{VE}) ; & \check{p}\left[\bar{v}_{p}\left(\bar{\nu}_{m}^{p}\right), \bar{u}_{p}\left(\bar{\nu}_{m}^{p}\right)\right]=0 .\end{cases}
$$

The variables $\bar{u}_{p}, \bar{v}_{p}$ are given by Eqs. (110) and (111). To avoid complex notation in the time domain, we define functions $\bar{R}_{x}^{p}, \bar{R}_{y}^{p}$ as

$$
\begin{aligned}
& \bar{R}_{x}^{p}(k)=\frac{\kappa}{k} \frac{\check{s}\left(\bar{v}_{p}, \bar{w}_{p}\right) \check{s}\left(\bar{w}_{p}, \bar{u}_{p}\right)}{\check{s}^{\prime}\left(\bar{v}_{p}, \bar{u}_{p}\right)}, \\
& \bar{R}_{y}^{p}(k)=\beta_{p}^{2} \frac{\kappa}{k} \frac{\check{p}\left(\bar{v}_{p}, \bar{w}_{p}\right) \check{p}\left(\bar{w}_{p}, \bar{u}_{p}\right)}{\check{p}^{\prime}\left(\bar{v}_{p}, \bar{u}_{p}\right)},
\end{aligned}
$$

which are real for $k \in \mathbb{R}$ and satisfy

$$
\begin{aligned}
& \bar{R}_{x}^{p}(k)+R_{x}^{p}(i k)=0, \\
& \bar{R}_{y}^{p}(k)+R_{y}^{p}(i k)=0 .
\end{aligned}
$$

In addition, as it is shown in Appendix $\mathrm{C}, R_{x, y}^{p}(k)$ and
$\bar{R}_{x, y}^{p}(k)$ are even functions. Similar to Eqs. (146), $\bar{R}_{x}^{p}, \bar{R}_{y}^{p}$ on the imaginary poles are given by

$$
\begin{aligned}
& \bar{R}_{x}^{p}\left(\bar{\mu}_{m}^{p}\right)=+\frac{3 \pi}{2} \frac{\check{s}^{2}\left(\bar{w}_{p}, \bar{u}_{p}\right)}{\bar{u}_{p} \frac{d \bar{u}_{p}}{d \kappa}-\bar{v}_{p} \frac{d \bar{v}_{p}}{d \kappa}\left\{\frac{\mathrm{Bi}^{\prime}\left(\bar{u}_{p}\right)}{\mathrm{Bi}^{\prime}\left(\bar{v}_{p}\right.}\right)^{2}}, \\
& \bar{R}_{y}^{p}\left(\bar{\nu}_{m}^{p}\right)=-\frac{3 \pi}{2} \frac{\beta_{p}^{2} \check{p}^{2}\left(\bar{w}_{p}, \bar{u}_{p}\right)}{\frac{d \bar{u}_{p}}{d \kappa}-\frac{d \bar{v}_{p}}{d \kappa}\left\{\frac{\operatorname{Bi}\left(\bar{u}_{p}\right)}{\operatorname{Bi}\left(\bar{v}_{p}\right)}\right\}^{2}},
\end{aligned}
$$

where the variables $\bar{u}_{p}, \bar{v}_{p}, \bar{w}_{p}, \beta_{p}$, and $\kappa$ on the right-hand sides are evaluated at $k=\bar{\mu}_{m}$ or $\bar{\nu}_{m}$, respectively. $\bar{R}_{x}^{p}\left(\bar{\mu}_{m}^{p}\right)$, $\bar{R}_{y}^{p}\left(\bar{\nu}_{m}^{p}\right)$ are positive for any $m$ and $p$.

According to the above, we finally obtain the longitudinal field of CSR in the time domain:

$$
\begin{aligned}
& \tilde{E}_{s}(z)=\frac{2 \pi q_{e}}{\varepsilon_{0} h} \int_{-\infty}^{\infty} d z^{\prime} \frac{d \tilde{\lambda}\left(z^{\prime}\right)}{d z^{\prime}} \Phi\left(z-z^{\prime}\right), \\
& \Phi(\zeta)=\sum_{p=0}^{\infty} \Lambda_{p}\left[2 \theta(-\zeta) J_{p}(\zeta)-K_{p}(\zeta)\right] .
\end{aligned}
$$

$J_{p}$ and $K_{p}$ are given by

$$
\begin{aligned}
& J_{p}(\zeta)=\sum_{m=1}^{\infty}\left[\frac{R_{x}^{p}\left(\mu_{m}^{p}\right)}{\mu_{m}^{p}} \sin \left(\mu_{m}^{p} \zeta\right)+\frac{R_{y}^{p}\left(\nu_{m}^{p}\right)}{\nu_{m}^{p}} \sin \left(\nu_{m}^{p} \zeta\right)\right], \\
& K_{p}(\zeta)=\sum_{m=1}^{\infty}\left[\frac{\bar{R}_{x}^{p}\left(\bar{\mu}_{m}^{p}\right)}{\bar{\mu}_{m}^{p}} e^{-\bar{\mu}_{m}^{p}|\zeta|}+\frac{\bar{R}_{y}^{p}\left(\bar{\nu}_{m}^{p}\right)}{\bar{\nu}_{m}^{p}} e^{-\bar{\nu}_{m}^{p}|\zeta|}\right] .
\end{aligned}
$$

Although Eq. (152) has an infinite sum with respect to the vertical Fourier mode $p$, the fundamental mode dominates the sum. In a square pipe, for example, the field magnitude of each vertical Fourier mode is of the order of $10^{-3}$ for $p=3$, and $10^{-5}$ for $p=4$ against the fundamental mode. It is therefore sufficient to take the terms to $p=2$ or 3 in the sum. If $\gamma=\infty$, since the space charge effect dies out, one can put $\Lambda_{p}=1$ in Eq. (153). Furthermore, if $x_{a}=$ $-x_{b}$ and $\gamma=\infty$, we have $\bar{\mu}_{m}^{p}=\mu_{m}^{p}$ for the HE mode and $\bar{\nu}_{m}^{p}=\nu_{m}^{p}$ for the VE mode. In this special case, $R_{x, y}^{p}$ and $\bar{R}_{x, y}^{p}$ have a simpler relation:

$$
\begin{aligned}
& \bar{R}_{x}^{p}(k)+R_{x}^{p}(k)=0, \\
& \bar{R}_{y}^{p}(k)+R_{y}^{p}(k)=0 .
\end{aligned}
$$

The longitudinal field has modes of two types: $J_{p}$ is an oscillatory mode, $K_{p}$ is a damped mode (evanescent mode). The oscillatory mode consists of the trigonometric functions, and because of the step function, this mode cannot overtake the source charge of $\gamma=\infty$. In contrast to the oscillatory mode, $K_{p}$ involving the exponential functions is a damped mode that can catch up the source charge; the field always clings to the bunch and stays within a short distance. 
As shown in Appendix A, the exact impedance, given by Eq. (A1), has singularities at $|k|=\alpha_{p}$, and the poles reach the singular points for infinitely wide chamber. In the parallel plates model, correspondingly, the exponential factors of the damped mode become

$$
e^{-\bar{k}_{m}|\xi|} \rightarrow e^{-\alpha_{p}|\xi|}
$$

This exponential field decay between infinite parallel plates was predicted, i.e., the fundamental vertical mode $e^{-\alpha_{0}|\zeta|}=e^{-\pi|\zeta / h|}$ agrees with Eq. (5.34b) of [5] which was observed by considering the asymptotic behavior of the longitudinal field at large distance in front of the source charge. In reality, the damped mode of Eq. (155) is correct regardless of the longitudinal distance between the observer and the charge.

\section{Comparison with simulation}

Figure 11 shows the longitudinal field in the time domain given by Eq. (152). We used a Newton search to obtain the pole values. The analytical solution (solid line) agrees with the numerical solution (small circles) obtained by our grid simulation using the paraxial approximation $[11,12]$. The fine difference between them is due to the transient state in the simulation, i.e., the field is still fluctuating a little bit in the simulation. As the figure shows, if we do not consider the imaginary poles (dashed line), the

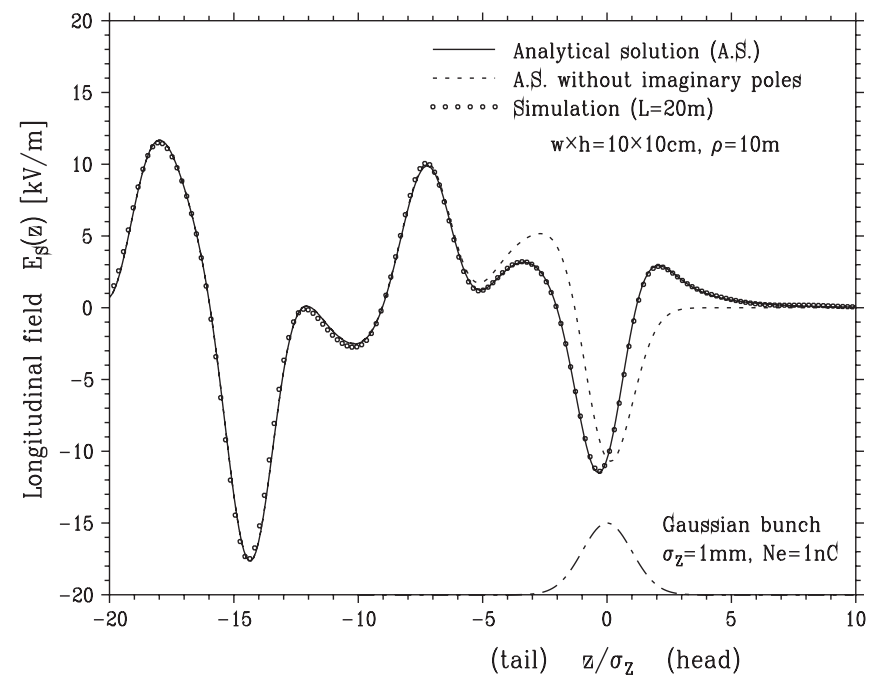

FIG. 11. The longitudinal field of CSR in a square pipe. The horizontal axis is the longitudinal position in the Gaussian bunch in units of the rms bunch length $\sigma_{z}=1 \mathrm{~mm}$. The dot-dashed line is the Gaussian bunch. The bunch charge is $q_{e}=+1 \mathrm{nC}$ (positron), the Lorentz factor is $\gamma=\infty$. The solid line represents the analytical solution of Eq. (152), the small circles indicate the simulation result in which we set the magnet length to $20 \mathrm{~m}$ so that the field becomes nearly stationary in a range of $|z|<20 \sigma_{z}$. The dashed line is the analytical solution of Eq. (152) without $K_{p}$ of Eq. (155). The chamber cross section is $x_{b}=-x_{a}=5 \mathrm{~cm}$ and $h=10 \mathrm{~cm}$, and the bending radius is $\rho=10 \mathrm{~m}$. analytical solution does not agree with the simulation result, and the damped mode coming from the imaginary poles must be taken into account. Figure 12 compares the field in a rectangular pipe with a parallel plates model. As we described in Sec. III B, if $w>2 h$, the field in the pipe is similar to that of parallel plates.

The Fourier transform of Eq. (67) is given as follows:

$$
\tilde{E}_{s}(z)=-q_{e} \int_{-\infty}^{\infty} W\left(z-z^{\prime}\right) \tilde{\lambda}\left(z^{\prime}\right) d z^{\prime} .
$$

Accordingly, integration by parts for Eq. (152) gives the longitudinal wakefield of CSR:

$$
W(\zeta)=-\frac{2 \pi}{\varepsilon_{0} h} \sum_{p=0}^{\infty} \Lambda_{p}\left[2 \theta(-\zeta) U_{p}(\zeta)+\operatorname{sgn}(\zeta) V_{p}(\zeta)\right]
$$

$$
U_{p}(\zeta)=\sum_{m=1}^{\infty}\left[R_{x}^{p}\left(\mu_{m}^{p}\right) \cos \left(\mu_{m}^{p} \zeta\right)+R_{y}^{p}\left(\nu_{m}^{p}\right) \cos \left(\nu_{m}^{p} \zeta\right)\right]
$$

$$
V_{p}(\zeta)=\sum_{m=1}^{\infty}\left[\bar{R}_{x}^{p}\left(\bar{\mu}_{m}^{p}\right) e^{-\bar{\mu}_{m}^{p}|\zeta|}+\bar{R}_{y}^{p}\left(\bar{\nu}_{m}^{p}\right) e^{-\bar{\nu}_{m}^{p}|\zeta|}\right] .
$$

Equation (159) depicts the longitudinal field of the opposite sign at position $z$ generated by a unit point charge at $z^{\prime}$.

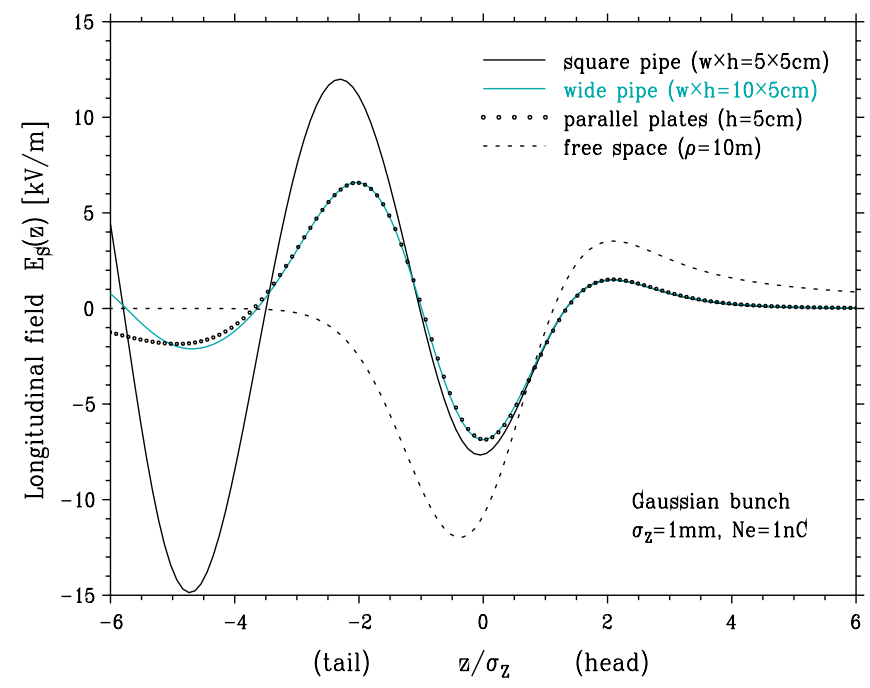

FIG. 12. (Color) Comparison of rectangular pipe with parallel plates. The black solid line represents the field in a rectangular pipe of $w \times h=5 \mathrm{~cm} \times 5 \mathrm{~cm}$; the cyan solid line is for a wide pipe of $w \times h=10 \mathrm{~cm} \times 5 \mathrm{~cm}$. They are obtained from Eq. (152). The small circles show the field between parallel plates with a spacing $h=5 \mathrm{~cm}$, which is computed by a numerical Fourier transform for Eq. (76). The dashed line is the longitudinal field in free space, given by Eq. (83). The bunch is Gaussian with a length $\sigma_{z}=1 \mathrm{~mm}$, and the charge is $q_{e}=$ $+1 \mathrm{nC}$. The bending radius is $\rho=10 \mathrm{~m}$, and the Lorentz factor is $\gamma=\infty$. 
Notice that the damped mode describes a direct interaction between two particles on the curved orbit. Denoting the longitudinal force due to the damped mode as $\tilde{F}_{s}^{\mathrm{D}} \propto$ $\operatorname{sgn}(\zeta) V_{p}(\zeta)$, we can decompose it into two terms:

$$
\tilde{F}_{s}^{\mathrm{D}}(\zeta) \propto \theta(\zeta) e^{-\bar{k}_{m} \zeta}-\theta(-\zeta) e^{\bar{k}_{m} \zeta} .
$$

Equation (162) says that, when the test particle (position $z$ ) is ahead of the source particle of the field (position $z^{\prime}$ ), the test particle is accelerated (i.e., forward force), and vise versa. That is, $\tilde{F}_{s}^{\mathrm{D}}$ is a repulsive force which follows the law of action and reaction: $\tilde{F}_{s}^{\mathrm{D}}(-\zeta)=-\tilde{F}_{s}^{\mathrm{D}}(\zeta)$. Therefore, the damped mode does not give rise to a net energy change within a bunch. Figure 13 shows the damped mode and the oscillatory mode. Considering a longitudinal charge distribution, the damped mode is determined by the balance of the source charges between the front and back with respect to the observation point. Accordingly, for a Gaussian bunch, the exponential force is zero at the bunch center. In this regard, the damped mode somewhat resembles a picture of usual space charge field on a straight orbit, while it has a different dependence on the distance and on the energy.

We would like to discuss Eq. (38) in [10], which is the longitudinal wakefield for a single resonance mode of CSR, induced by a single particle in a curved rectangular waveguide. In our notation it is written as

$$
w_{m}(\zeta)=2 x_{m} \cos \left(k_{m} \zeta\right)
$$

where $x_{m}$ is a loss factor due to the impedance of CSR. Superposition of $w_{m}$ for all resonance modes gives the

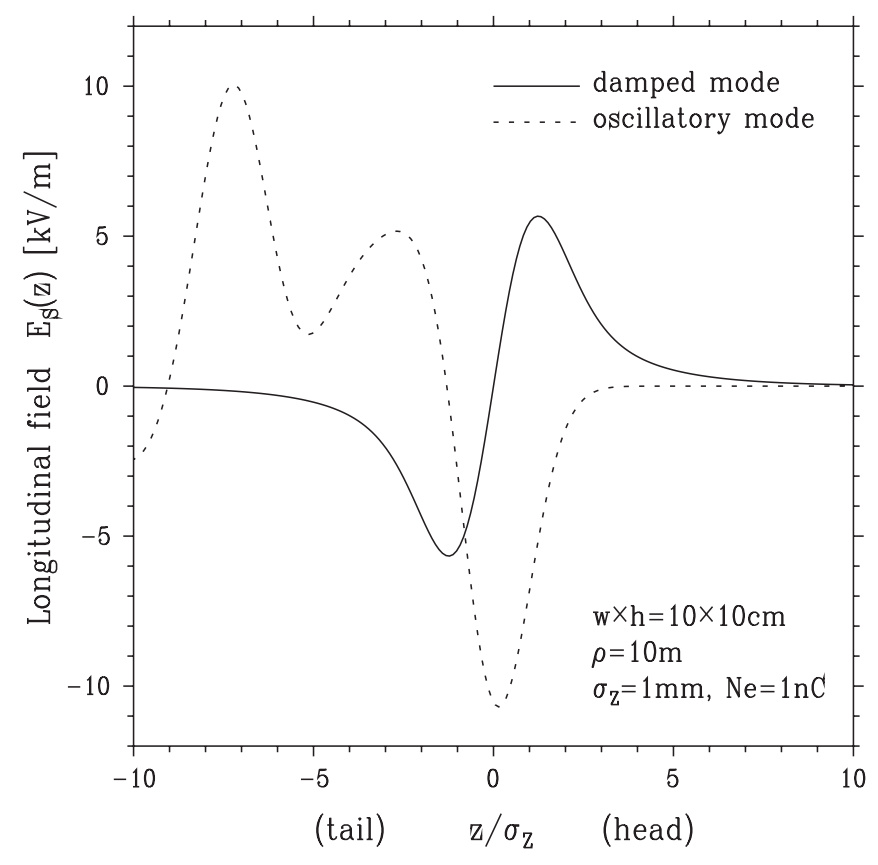

FIG. 13. The longitudinal field of the damped mode (solid line) and the oscillatory mode (dashed line) for a Gaussian bunch. The parameters are the same as Fig. 11. longitudinal wakefield, i.e.,

$$
W(\zeta)=\sum_{m=1}^{\infty} w_{m}(\zeta)
$$

Considering the field causality, to be precise, Eq. (163) should have $\theta(-\zeta)$, or the integration limit of Eq. (158) should be $z^{\prime}=[z, \infty]$ for $w_{m}$. Equation (163) corresponds to Eq. (160); therefore the loss factor is obtained as

$$
\varkappa_{m}=-\frac{2 \pi}{\varepsilon_{0} h} \sum_{p=0}^{\infty}\left[R_{x}^{p}\left(\mu_{m}^{p}\right)+R_{y}^{p}\left(\nu_{m}^{p}\right)\right],
$$

which is also given as a resonance mode of Eq. (141) for a unit point charge. However, Eq. (163) does not have the damped mode of Eq. (161); the wakefield given by Eq. (38) of [10] is therefore incomplete. CSR can overtake the field source because the orbit is curved; one cannot apply the usual wake-impedance relation for a straight orbit such as in Eqs. (2.85) and (2.86) of [18]. The loss factor of Eq. (165) is still correct, because the damped mode describes the interaction among the particles within the bunch and thereby has no contribution to the loss factor.

\section{APPLICATION LIMIT OF THE PARAXIAL APPROXIMATION}

This section describes the conversion of the real impedance given by Eq. (139) from rectangular pipe to infinite parallel plates. This procedure is instructive to examine the limits of the paraxial approximation. This conversion does not agree with the assumption that the pipe width is much smaller than the bending radius of the dipole magnet, i.e., $\sqrt{w / \rho} \ll 1$ in [10-12]; however, we will show that the impedance is still correct.

The real impedance of Eq. (139) contains the field damping due to an infinitesimal resistivity or energy leak of the pipe; therefore we can just move the sidewalls away without a particular treatment. To obtain the approximate resonance poles on the real axis, let us rederive the results of previous authors $[2,3,10]$.

\section{A. Influence of the sidewalls}

At first, we will estimate the wave number which is not affected by the sidewalls. For large real wave numbers, the Airy functions involving the inner wall behave as

$$
\begin{gathered}
\operatorname{Ai}\left(u_{p}\right), \operatorname{Ai}^{\prime}\left(u_{p}\right) \propto e^{-\zeta_{u},} \\
\operatorname{Bi}\left(u_{p}\right), \operatorname{Bi}^{\prime}\left(u_{p}\right) \propto e^{+\zeta_{u},} \\
\zeta_{u}=(2 / 3) u_{p}^{3 / 2} \in \mathbb{R} .
\end{gathered}
$$

$u_{p}$ is defined in Eq. (93). For simplicity, we put $\gamma=\infty$ in discussing the shielding effect. Equations (166)-(168) give the condition that makes the inner wall invisible: 


$$
2 \zeta_{u} \gg 1
$$

namely,

$$
\frac{2}{3} k \rho\left[\left(\frac{\alpha_{p}}{k}\right)^{2}+\frac{-2 x_{a}}{\rho}\right]^{3 / 2} \gg 1 .
$$

$\zeta_{u}$ has a minimal value $\zeta_{u}^{\min }$ at $k=k_{\min }$ :

$$
\begin{gathered}
k_{\min }=\alpha_{p} \sqrt{\frac{\rho}{-x_{a}}}, \\
\zeta_{u}^{\min }=\sqrt{3} \alpha_{p}\left(-x_{a}\right) .
\end{gathered}
$$

The lowest vertical mode dominates the vertical Fourier series of the field, therefore if

$$
-x_{a} \gg \frac{h}{2 \pi \sqrt{3}},
$$

then Eq. (170) is satisfied for $\forall k>0$. As we discussed in Sec. IV, the outer wall has the same mathematical structure as the inner wall. Accordingly, if the beam is running at the center of the pipe, we have

$$
\frac{w}{h} \gg \frac{1}{\pi \sqrt{3}} .
$$

Equation (174) roughly agrees with Eq. (91) which is the condition that makes the sidewalls negligible in the low frequency limit. That is, it is the condition where the parallel plates impedance agrees with that of rectangular pipe over the whole range of wave numbers.

Let us next consider the opposite case, i.e., $w \ll h / \pi$, which is unusual in a dipole magnet of accelerators. Under this assumption, the first term in the bracket of Eq. (170) is much smaller than the second term; therefore the wave number which satisfies Eq. (170) is given by

$$
k \gg \frac{3}{2} \sqrt{\frac{\rho}{\left(-2 x_{a}\right)^{3}}} \equiv k_{\mathrm{th}}^{(\mathrm{in})} .
$$

$k_{\mathrm{th}}^{(\mathrm{in})}$ is the criterion for the inner wall in the absence of the upper-lower plates $(h \rightarrow \infty)$. If the beam travels at the center of the cylindrical sidewalls, the criterion above which the field does not see the sidewalls is

$$
k_{\mathrm{th}}^{(\text {side })}=\frac{3}{2} \sqrt{\frac{\rho}{w^{3}}} .
$$

As it is understood from the parallel plates impedance of Eq. (76), the shielding threshold of the parallel plates, Eq. (2), is derived by solving

$$
\zeta_{w}=(2 / 3) w_{p}^{3 / 2} \gg 1,
$$

with $\gamma=\infty$. From Eq. (177), we actually obtain

$$
k \gg \sqrt{\frac{\rho}{3} \alpha_{p}^{3}} \simeq \pi \sqrt{\frac{\rho}{h^{3}}}=k_{\mathrm{th}} .
$$

In accordance with the condition of Eq. (169), one can use $2 \zeta_{w} \gg 1$ instead of Eq. (177) to obtain the shielding threshold. In this definition the coefficient of the threshold wave number will change by a factor of $\sqrt{2}$ :

$$
k_{\mathrm{th}}^{\dagger}=\sqrt{\frac{2 \rho}{3}\left(\frac{\pi}{h}\right)^{3}} .
$$

The real part of the parallel plates impedance behaves as $\Re Z(k) \propto e^{-\left(k_{\mathrm{th}}^{\dagger} / k\right)^{2}}$ below $k_{\mathrm{th}}^{\dagger}$. Regarding the shielding effect, Eqs. (176) and (179) imply the asymmetry between the chamber width and the height:

$$
\frac{2 \pi}{3} w \leftrightarrow h .
$$

The factor $2 \pi / 3$ may indicate the asymmetric ratio of the lowest contour at $-2000 \mathrm{eV}$ (i.e., $w \gg h$ or $w \ll h$ ) in Fig. 17 of [15] which shows an energy change due to CSR for various chamber sizes. For arbitrary $w$ and $h$, one can obtain the shielding threshold by solving Eq. (170) using Cardano's formula for the cubic. The solution is, however, considerably involved due to the influence both from the upper-lower walls and the sidewalls.

\section{B. Solution of the real poles}

At high frequency such that the inner wall is invisible, $k \gg k_{\mathrm{th}}^{\text {(in) }}$, the Airy functions $\mathrm{Ai}\left(u_{p}\right)$ and $\mathrm{Ai}^{\prime}\left(u_{p}\right)$ are exponentially small as shown in Eqs. (166); then the cross products behave for large real wave numbers as

$$
\begin{aligned}
& \check{s}\left(v_{p}, u_{p}\right) \simeq \operatorname{Ai}^{\prime}\left(v_{p}\right) \operatorname{Bi}^{\prime}\left(u_{p}\right), \\
& \check{p}\left(v_{p}, u_{p}\right) \simeq \operatorname{Ai}\left(v_{p}\right) \operatorname{Bi}\left(u_{p}\right) .
\end{aligned}
$$

Since $\operatorname{Bi}\left(u_{p}\right)$ and $\operatorname{Bi}^{\prime}\left(u_{p}\right)$ have no zeros on the real $k$ axis, the approximate resonance conditions are

$$
\begin{aligned}
& \mathrm{Ai}^{\prime}\left[v_{p}\left(\mu_{m}\right)\right]=0 \quad \text { HE mode, } \\
& \operatorname{Ai}\left[v_{p}\left(\nu_{m}\right)\right]=0 \quad \text { VE mode. }
\end{aligned}
$$

These are resonance conditions for the pillbox chamber of Eq. (71) in itself. At the resonance points of the HE mode, the cross products involving derivatives of the Airy functions become as follows:

$$
\begin{aligned}
& \check{q}\left[v_{p}\left(\mu_{m}\right), u_{p}\left(\mu_{m}\right)\right] \simeq \operatorname{Ai}\left[v_{p}\left(\mu_{m}\right)\right] \operatorname{Bi}^{\prime}\left[u_{p}\left(\mu_{m}\right)\right], \\
& \check{r}\left[v_{p}\left(\mu_{m}\right), u_{p}\left(\mu_{m}\right)\right] \simeq 0 .
\end{aligned}
$$

On the other hand, at the VE resonances, we have

$$
\begin{aligned}
& \check{q}\left[v_{p}\left(\nu_{m}\right), u_{p}\left(\nu_{m}\right)\right] \simeq 0, \\
& \check{r}\left[v_{p}\left(\nu_{m}\right), u_{p}\left(\nu_{m}\right)\right] \simeq \operatorname{Ai}^{\prime}\left[v_{p}\left(\nu_{m}\right)\right] \operatorname{Bi}\left[u_{p}\left(\nu_{m}\right)\right] .
\end{aligned}
$$

Expanding $\operatorname{Ai}\left(v_{p}\right)$ and $\operatorname{Ai}^{\prime}\left(v_{p}\right)$ in their asymptotic series,

$$
\operatorname{Ai}^{\prime}\left(v_{p}\right) \simeq-\frac{\left(-v_{p}\right)^{1 / 4}}{\sqrt{\pi}} \cos \left(\zeta_{v}+\frac{\pi}{4}\right),
$$




$$
\begin{aligned}
\operatorname{Ai}\left(v_{p}\right) & \simeq \frac{\left(-v_{p}\right)^{-1 / 4}}{\sqrt{\pi}} \sin \left(\zeta_{v}+\frac{\pi}{4}\right), \\
\zeta_{v} & =(2 / 3)\left(-v_{p}\right)^{3 / 2} \in \mathbb{R},
\end{aligned}
$$

the resonance conditions are approximately given by

$$
\zeta_{v}=\pi(m-d) \quad(m=1,2,3, \ldots) .
$$

$d$ is a constant of Eq. (99) and can be dropped when the mode index $m$ is large. From Eqs. (187) and (188), we obtain a cubic equation:

$$
Y^{3}+b Y-1=0,
$$

where we put

$$
\begin{gathered}
Y=\frac{1}{\kappa}\left(\frac{\alpha_{p}^{2}}{x_{b}}\right)^{1 / 3}, \\
b=\left(\frac{3 \zeta_{v}}{2 \alpha_{p} x_{b}}\right)^{2 / 3} .
\end{gathered}
$$

$\alpha_{p}$ is the $p$ th vertical wave number given by Eq. (25); the fundamental mode, $\alpha_{0}=\pi / h$, dominates the impedance value in the vertical Fourier modes. Using Cardano's formula to solve the cubic, we get the approximate resonance points on the real wave number axis, i.e.,

$$
k_{m}=\alpha_{p} \sqrt{\frac{\rho}{x_{b}}} \Upsilon\left(\frac{h(m-d)}{x_{b}(2 p+1)}\right),
$$

where $Y(r)$ is a function defined by Eq. (100).

\section{Condition of the paraxial approximation}

Returning to Eq. (189), we can neglect the term of $Y^{3}$ in Eq. (189) under the condition

$$
\sqrt{\frac{2 x_{b}}{\rho}} \gg \frac{\alpha_{p}}{k},
$$

then the resonance points become equally spaced:

$$
\begin{aligned}
& k_{m}=(m-d) \Delta \hat{k}, \\
& \Delta \hat{k}=3 \pi \sqrt{\frac{\rho}{\left(2 x_{b}\right)^{3}}} .
\end{aligned}
$$

Provided that Eq. (193) holds also in the shielded region, $k<k_{\text {th }}$, the pipe width must satisfy

$$
w \gg \rho\left(\frac{\alpha_{p}}{k_{\mathrm{th}}}\right)^{2} .
$$

Since the fundamental mode is dominant in the vertical Fourier modes, Eq. (196) is equivalent to Eq. (91), which means $k_{1} \ll k_{\text {th }}$ for Eq. (194). That is, if the lowest resonance occurs well below the shielding threshold of the upper-lower walls, the sidewalls are negligible. As we have shown in Sec. III B, if the aspect ratio of the pipe is large, $w \gg h / \pi$, the low frequency components of the field are suppressed almost only by the upper-lower walls and do not depend on the chamber width. Therefore if the aspect ratio is large, we do not need to assume $\sqrt{w / \rho} \ll 1$, i.e., the chamber width can be infinitely large in the paraxial approximation if we keep $\sqrt{h / \rho} \ll 1$. Owing to the characteristics of the shielded CSR, the paraxial approximation can deal with the infinite parallel plates in the range

$$
k \gg \frac{\pi}{h},
$$

where the shielding effect by the upper-lower plates is considered.

On the other hand, at very high frequency, $k \gg k_{\mathrm{th}}$, as the wall effect vanishes, the chamber height does not matter and can be very large. If $h=\rho$, for example, the lowest vertical wave number is equal to the threshold wave number of shielding: $\alpha_{0}=k_{\mathrm{th}}$. In this case, the valid wave number range is given by

$$
k \gg \frac{\pi}{\rho} .
$$

In fact, by taking the limit as $h \rightarrow \infty$, we obtained the free space impedance of Eq. (79) in the framework of the paraxial approximation. In contrast, the wakefield of Eq. (83) was originally derived in the time domain [6,7], where the bunch length is assumed to be much shorter than the bending radius. This condition is equivalent to $k \rho \gg 1$ and consistent with the condition of the uniform asymptotic expansion, shown in Appendix A. According to the above discussion, the paraxial approximation works in the following wave number range:

$$
k \gg \frac{\pi}{\min [w, h, \rho]} .
$$

This is the condition which we assumed at (c) of Sec. II A. To be precise, it is better to replace $w$ in Eq. (199) by $(2 \pi / 3) w$. Usually, one has $h \leq w \ll \rho$ in accelerators; therefore, the paraxial approximation is applicable if

$$
\pi \frac{\sigma_{z}}{h} \ll 1 .
$$

Then the paraxial approximation has a relative error of the order of $\left(\pi \sigma_{z} / h\right)^{2}$. The assumption that $\sqrt{h / \rho} \ll 1$ is equivalent to $\alpha_{0} \ll k_{\mathrm{th}}$ which is needed to extend the valid range of the wave number well below the shielding threshold in order to take the wall effect into account. If a bunch length is similar to the chamber height, the paraxial approximation breaks down. However, such a long bunch is usually not affected by CSR, and thus there is no need to estimate it.

One has another thing to consider at very low frequency; that is the resistive wall effect. Although in this paper, we have assumed a perfectly conducting pipe, in reality, as Fig. 6 shows, the resistive wall impedance is comparable to 
CSR impedance at low frequency $k \sim \pi / h$. Then CSR is overwhelmed by the resistive wall effect in $k<\pi / h$. That is, the assumption of perfectly conducting walls also breaks down at very low frequency.

\section{Real impedance and power spectrum}

Let us go back to the previous discussion about the conversion of the impedance to that of parallel plates. From Eqs. (183) and (184), we have

$$
\begin{aligned}
& R_{x}^{p}\left(\mu_{m}\right)=\left.\frac{3 \mathrm{Ai}^{12}\left(w_{p}\right)}{2 v_{p}^{\prime}\left(-v_{p}\right)^{1 / 2}}\right|_{k=\mu_{m}}, \\
& R_{y}^{p}\left(\nu_{m}\right)=\left.\frac{3 \beta_{p}^{2} \operatorname{Ai}^{2}\left(w_{p}\right)}{2 v_{p}^{\prime}\left(-v_{p}\right)^{1 / 2}}\right|_{k=\nu_{m}},
\end{aligned}
$$

where $v_{p}^{\prime}=d v_{p} / d \kappa$. If the aspect ratio of the pipe is high, as the resonance mode index $m$ becomes very large for a given wave number, eventually, the resonance points of the HE mode agree with the VE resonances, i.e., $k_{m}=\mu_{m}=$ $\nu_{m}$ in the limit $x_{b} \rightarrow \infty$. Then $k_{m}$ becomes

$$
k_{m}=m \Delta \hat{k} \text {. }
$$

Accordingly, the real impedance of Eq. (139) reduces to

$$
\frac{\Re Z(k)}{Z_{0}}=\frac{2 \pi}{\beta h}\left(\frac{2}{k \rho}\right)^{1 / 3} M(k) \sum_{p=0}^{\infty} \Lambda_{p} \Re F_{0}\left(\beta_{p}\right),
$$

where $M$ is a sum with respect to the resonance mode:

$$
M(k)=\sum_{\substack{m=-\infty \\ m \neq 0}}^{\infty} \delta\left(k-k_{m}\right) \Delta \hat{k} .
$$

In the limit of $x_{b} \rightarrow \infty$, the resonance interval $\Delta \hat{k}$ is infinitesimal; the impedance becomes a continuous function with respect to the wave number. Consequently, $M$ becomes an integral:

$$
M=\int_{-\infty}^{\infty} \delta(k-\hat{k}) d \hat{k}=1,
$$

and then Eq. (203) agrees with the real part of Eq. (76). As we have shown in Sec. III A, the real impedance can be derived also from the imaginary impedance of Eq. (68) by taking the limit $x_{a} \rightarrow-\infty$ and $x_{b} \rightarrow \infty$ with an infinitesimal damping; $k=k+i \epsilon(\epsilon \rightarrow+0)$.

Stupakov and Kotelnikov derived the power spectrum of synchrotron radiation as follows [10]. Using Vainshtein's theory, the longitudinal electric field is described with the group velocity which is calculated from the Poynting vector and the energy density of the field. Considering the energy loss rate in the high frequency limit, the power spectrum is obtained. Since we have the impedance, the total radiated power per unit frequency per revolution is obtained using

$$
\frac{d P}{d \omega}=2 \beta \rho\left|q_{e} \lambda(k)\right|^{2} \Re Z(k),
$$

where we are considering a finite $\gamma$. Equation (206) is correct only for positive real $k$. We now assume that the field source is a single point charge of charge $e: q_{e} \lambda(k)=$ $e$. We put $t=\beta_{p}$ and $b_{c}=k / \kappa \gamma$ in Eq. (203) with $M=1$ and consider the free space impedance. For $h \rightarrow \infty$, similar to Eq. (78), the sum with respect to the vertical Fourier mode becomes the following integral:

$$
\begin{aligned}
\frac{d P}{d \omega} & =Z_{0} e^{2}(4 k \rho)^{1 / 3} \int_{-\infty}^{\infty} \Re F_{0}(t) d t, \\
& =-Z_{0} e^{2}(k \rho)^{1 / 3}\left(\operatorname{Ai}^{\prime}\left(z_{c}\right)+\frac{b_{c}^{2}}{2^{1 / 3}} \int_{z_{c}}^{\infty} \mathrm{Ai}(z) d z\right), \\
& =\frac{Z_{0} e^{2}}{2 \pi \sqrt{3}} \frac{k \rho}{\gamma^{2}}\left(2 K_{2 / 3}\left(k / k_{c}\right)-\int_{k / k_{c}}^{\infty} K_{1 / 3}(x) d x\right), \\
& =\frac{\sqrt{3}}{4 \pi} Z_{0} e^{2} \gamma \frac{k}{k_{c}} \int_{k / k_{c}}^{\infty} K_{5 / 3}(x) d x,
\end{aligned}
$$

where $z_{c}=\left(3 k / 2 k_{c}\right)^{2 / 3}, k_{c}$ is the critical wave number, i.e., $k_{c}=3 \gamma^{3} / 2 \rho$. Thus, from the radiation impedance, one can obtain the power spectrum of incoherent synchrotron radiation for a single particle.

\section{CONCLUSION}

In the paraxial approximation we have obtained the analytical expression of the longitudinal electric field of steady CSR in the time domain, which is propagating in a perfectly conducting rectangular pipe of constant curvature. The solution is in agreement with our simulation results using the paraxial approximation.

We have shown that the field consists of an oscillatory mode and a damped mode. The oscillatory mode is represented by the resonance poles on the real wave number axis and composed by superposing sinusoidal waves at the resonance points. The oscillatory mode is always delayed from the source charge and hence the field has a long tail behind the bunch similar to usual wakefields on a straight orbit. On the other hand, the damped mode is brought by the imaginary poles of the impedance; this field can exist also in front of the bunch; however, because of the exponential decay, it always goes along with the source charge and does not behave like a wave. This evanescent mode forms a field of repulsive force between two particles; the delayed particles can affect the leading particles through this damped mode. These two kinds of modes have a different dependence on the sidewalls of the pipe: the outer wall dominates the oscillatory mode especially in the high frequency; in contrast, the inner wall mainly determines the damped mode around the bunch.

The asymptotic expansion of the impedance at low frequency reveals that the paraxial approximation properly considers the space charge field, though the effect is very small at high energy: $\gamma \gg k h / \pi$. The asymptotic expression also implies that the upper-lower walls of the beam 
pipe are more effective at shielding CSR than the sidewalls. The condition that makes the sidewalls negligible is $w \gg h / \pi$ which is about $w>2 h$ in practice.

Our theory is based on the paraxial approximation, so our formalism is correct provided that the longitudinal wave number is much larger than the vertical wave number: $k \gg \pi / h$, and the relative error is of the order of $(\pi / h k)^{2}$. The paraxial approximation is therefore applicable for $\sigma_{z} \ll h / \pi$. In addition, the assumption of perfectly conducting walls also breaks down in the very low frequency region: $k \leqq \pi / h$. However, if the main frequency components of a bunch spectrum are around the vertical cutoff wave number, owing to the shielding effect by the vacuum chamber, CSR would not cause a serious issue on the bunch, unless the bunch charge is extraordinarily large.

\section{ACKNOWLEDGMENTS}

We would like to sincerely acknowledge E. Forest for his valuable comments, suggestions, and proofreading of our draft. We wish to thank KEK for providing a desk, a chair, and computers free of charge. We would also like to thank $\mathrm{K}$. Yokoya for discussing this problem.

\section{APPENDIX A: UNIFORM ASYMPTOTIC EXPANSION FOR WARNOCK-MORTON'S THEORY}

This section describes the connection between our theory and Warnock-Morton's theory [2,4]. In this paper we derived three longitudinal impedances of CSR: i.e., rectangular pipe [Eq. (69)]; pillbox chamber [Eq. (71)]; and infinite parallel plates [Eq. (76)]. One finds that they are similar to the ones derived by Warnock and Morton. The torus impedance per revolution is given by

$$
\frac{Z(n, \omega)}{n}=i Z_{0} \frac{2 \pi^{2} \rho}{\beta h} \sum_{p=0}^{\infty} \Lambda_{p}\left(\beta^{2} G_{x}^{n}+\frac{\alpha_{p}^{2}}{\gamma_{p}^{2}} G_{y}^{n}\right),
$$

with

$$
\begin{aligned}
G_{x}^{n} & =\frac{s_{n}\left(\gamma_{p} r_{b}, \gamma_{p} \rho\right) s_{n}\left(\gamma_{p} \rho, \gamma_{p} r_{a}\right)}{s_{n}\left(\gamma_{p} r_{b}, \gamma_{p} r_{a}\right)}, \\
G_{y}^{n} & =\frac{p_{n}\left(\gamma_{p} r_{b}, \gamma_{p} \rho\right) p_{n}\left(\gamma_{p} \rho, \gamma_{p} r_{a}\right)}{p_{n}\left(\gamma_{p} r_{b}, \gamma_{p} r_{a}\right)},
\end{aligned}
$$

where $p_{n}$ and $s_{n}$ are cross products of Bessel functions of the first and the second kind,

$$
\begin{aligned}
p_{n}(x, y) & =J_{n}(x) Y_{n}(y)-Y_{n}(x) J_{n}(y), \\
s_{n}(x, y) & =J_{n}^{\prime}(x) Y_{n}^{\prime}(y)-Y_{n}^{\prime}(x) J_{n}^{\prime}(y) .
\end{aligned}
$$

$\alpha_{p}$ is the vertical wave number given by Eq. (25). $\gamma_{p}$ is the radial wave number:

$$
\gamma_{p}^{2}=(\beta k)^{2}-\alpha_{p}^{2}
$$

which is imaginary in $|k|<\alpha_{p} / \beta$. The parameters $r_{a}, r_{b}$ are the inner and the outer torus radii, respectively,

$$
\begin{aligned}
& r_{a}=\rho+x_{a}, \\
& r_{b}=\rho+x_{b} .
\end{aligned}
$$

If one defines the impedance at the center of the transverse beam position as we did, the form factor of the vertical beam distribution $\Lambda_{p}$ is given by Eq. (70). The impedance of Eq. (A1) looks similar to Eq. (69) but still differs. That is, the field has a periodic boundary condition along the orbit in their theory because of the circular motion of the beam; therefore, the field is composed of frequency components whose wavelengths are multiple integers of the orbital circumference:

$$
n=k \rho,
$$

where $n \in \mathbb{Z}$ is the harmonic number of the field along the circumference. The following figurative examples may illustrate the difference between their theory and ours. Imagine a recording hard disk; Warnock-Morton's theory writes data as fitting with the former data, while our theory just overwrites data as if there is nothing before. One can have another interpretation for our chamber model. The space has another dimension; the beam pipe stretches towards the extra dimension like a spiral staircase. As the bunch travels along the spiral pipe, it always emits radiation toward the extra dimension; hence, the new radiation has no interference with the previous fields. Because of such a chamber structure, in spite of the fact that the beam pipe is assumed to have infinite length with a constant curvature, rigorously speaking, our chamber model is not a torus; the field has no periodic boundary condition along the beam orbit. For removing the inner wall to obtain the pillbox impedance of Eq. (71) in Sec. III A, it is therefore necessary to take the limit: $x_{a} \rightarrow-\infty$ (not $x_{a} \rightarrow-\rho$ ).

Considering an actual accelerator, the wavelength of CSR is much shorter than the circumference of the ring; we do not have to consider the periodic boundary condition along the ring. It means in terms of mathematics that we can make a uniform asymptotic expansion (Olver expansion) for their impedance. That is, we can take the limit $n \rightarrow \infty$ along $n=x$ for $J_{n}(x), Y_{n}(x)$, and their derivatives. This expansion is applied in [2,3] to write a numerical code or to derive the approximate resonance points, which is shown in Sec. IVA. Let us derive our impedance of Eq. (69) from Eq. (A1). Applying the uniform asymptotic expansion for large $n$ given by 9.3.35-9.3.37 and 9.3.439.3.45 in [14]: 


$$
\begin{aligned}
& J_{n}(n z)=+g(z)\left[\frac{\mathrm{Ai}\left(n^{2 / 3} \zeta\right)}{n^{1 / 3}}+O\left(n^{-5 / 3}\right)\right], \\
& Y_{n}(n z)=-g(z)\left[\frac{\mathrm{Bi}\left(n^{2 / 3} \zeta\right)}{n^{1 / 3}}+O\left(n^{-5 / 3}\right)\right], \\
& J_{n}^{\prime}(n z)=-\frac{2}{z g(z)}\left[\frac{\mathrm{Ai}^{\prime}\left(n^{2 / 3} \zeta\right)}{n^{2 / 3}}+O\left(n^{-4 / 3}\right)\right], \\
& Y_{n}^{\prime}(n z)=+\frac{2}{z g(z)}\left[\frac{\mathrm{Bi}^{\prime}\left(n^{2 / 3} \zeta\right)}{n^{2 / 3}}+O\left(n^{-4 / 3}\right)\right],
\end{aligned}
$$

where $g(z)$ is

$$
g(z)=\left(\frac{4 \zeta}{1-z^{2}}\right)^{1 / 4}
$$

$z$ and $\zeta$ are not the longitudinal coordinates but variables which satisfy the following relation:

$$
\frac{2}{3}(+\zeta)^{3 / 2}=\log \frac{1+\sqrt{1-z^{2}}}{z}-\sqrt{1-z^{2}} \quad(z<1, \zeta>0)
$$

$\frac{2}{3}(-\zeta)^{3 / 2}=\sqrt{z^{2}-1}-\cos ^{-1}\left(\frac{1}{z}\right) \quad(z>1, \zeta<0)$.

The truncation in the uniform asymptotic series yields the relative error $\delta_{1}$ which is estimated by

$$
\delta_{1} \simeq \frac{c_{0}(\zeta)}{(k \rho)^{2 / 3}}
$$

where $c_{0}(\zeta)$ is given by 9.3 .46 of [14] and has a value of about $c_{0}(0)=2^{2 / 3} / 10 \approx 0.15874$. In deriving Eq. (7), the leading term we neglected is $O\left[(k \rho)^{-2 / 3}\right]$ which corresponds to $\delta_{1}$. In practice, the typical bunch length $\sigma_{z}$ (or structure size in the bunch) which concerns with harmful CSR is very short, i.e., the typical wave number is very large, $(k \rho)^{-2 / 3} \sim\left(\sigma_{z} / \rho\right)^{2 / 3} \ll 1$; therefore we can neglect the truncation error $\delta_{1}$.

The argument of the Bessel functions is

$$
n z=\gamma_{p}(\rho+x)
$$

the relevant variable $z$ is identified to

$$
z=\left(1+\frac{x}{\rho}\right) \sqrt{1-\frac{\alpha_{p}^{2}}{k^{2}}-\frac{1}{\gamma^{2}}}
$$

where $x$ represents $x_{a}, x_{b}$, or 0 for $r_{a}, r_{b}$, or $\rho$ in Eq. (A1). We assume that the vertical wave number is much smaller than the longitudinal one (paraxial approximation):

$$
\epsilon_{1}=\frac{\alpha_{p}}{k} \ll 1
$$

In addition, we assume the following things:

$$
\epsilon_{2}=\frac{1}{\gamma} \ll 1,
$$

$$
\epsilon_{3}=\sqrt{\frac{x}{\rho}} \ll 1
$$

Equation (A15) is a condition in deriving Eq. (7). One would think that it contradicts the limit $w \rightarrow \infty$. In practice, it is sufficient to consider a region for $x$ in which CSR exists: $x \sim \kappa^{-1}$ in free space, or $x \sim \alpha_{p}^{-1}$ between parallel plates. In other words, Eq. (A15) is equivalent to $1 / k \rho \ll$ 1 or $\sqrt{h / \rho} \ll 1$, respectively. So one can take the limit as $w \rightarrow \infty$ later with a virtual damping. $z$ is close to 1 under the assumptions from Eqs. (A13)-(A15),

$$
1-z^{2}=\epsilon_{1}^{2}+\epsilon_{2}^{2}-2 \epsilon_{3}^{2}+O\left(\epsilon_{i}^{4}\right)
$$

$\zeta$ is approximately obtained as

$$
\zeta=\frac{1-z^{2}}{2^{2 / 3}}\left\{1+\frac{2}{5}\left(1-z^{2}\right)+O\left[\left(1-z^{2}\right)^{2}\right]\right\},
$$

and the relative error is of the order of $O\left(\epsilon_{i}^{2}\right)$. In general, $z$ is a complex variable and changes from a real number to a purely imaginary number at $k= \pm \alpha_{p} / \beta$ where $\left(J_{n}, Y_{n}\right)$ are analytically continued into $\left(I_{n}, K_{n}\right)$. The real notation is switched from $\left(J_{n}, Y_{n}\right)$ to $\left(I_{n}, K_{n}\right)$ in $|k|<\alpha_{p} / \beta$. The asymptotic expression in the low frequency limit, given by Eq. (87), was derived with Debye expansion (9.7.79.7.10 of [14]) for $I_{n}, K_{n}$ [16].

The variables of the Airy functions are given by

$$
\begin{aligned}
n^{2 / 3} \zeta & =(k \rho / 2)^{2 / 3}\left(1-z^{2}\right)\left\{1+O\left(\epsilon_{i}^{2}\right)\right\}, \\
& \simeq w_{p}-\xi .
\end{aligned}
$$

$w_{p}$ is defined by Eq. (28); $\xi$ represents $\xi_{a}, \xi_{b}$, or 0 corresponding to $r_{a}, r_{b}$, or $\rho$. Then the cross products of the Bessel functions, $p_{n}(x, y), s_{n}(x, y)$, are expanded for large $n$ and become the Airy functions:

$$
\begin{aligned}
& \lim _{n \rightarrow \infty}(n / 2)^{2 / 3} p_{n}\left(\gamma_{p} r_{b}, \gamma_{p} r_{a}\right)=-\check{p}\left(v_{p}, u_{p}\right), \\
& \lim _{n \rightarrow \infty}(n / 2)^{4 / 3} s_{n}\left(\gamma_{p} r_{b}, \gamma_{p} r_{a}\right)=-\check{s}\left(v_{p}, u_{p}\right) / \beta^{2} .
\end{aligned}
$$

In the conditions of Eqs. (A13) and (A14), the radial wave number is close to the longitudinal wave number,

$$
\frac{\alpha_{p}^{2}}{\gamma_{p}^{2}}=\epsilon_{1}^{2}\left\{1+O\left(\epsilon_{1}^{2}, \epsilon_{2}^{2}\right)\right\}
$$

Taking the zeroth order term in Eq. (A21) yields the relative truncation error:

$$
\delta_{2}=\frac{\alpha_{p}^{2}}{k^{2}}+\frac{1}{\gamma^{2}},
$$

which determines the accuracy of the paraxial approximation, because the error $\delta_{1}$ of Eq. (A10) is usually smaller than $\delta_{2}$. In general, the paraxial approximation for classical electromagnetic waves is a zeroth order theory for Maxwell equations with respect to $\epsilon=k_{\perp} / k$; the formal- 
ism has a local error of the second order in $\epsilon$ [20], where $k_{\perp}$ is the transverse wave number of field and is equivalent to the inverse transverse expanse of the electromagnetic wave. CSR has a transverse wave number:

$$
k_{\perp}= \begin{cases}\kappa & \text { in free space, } \\
\alpha_{p} & \left\{\begin{array}{l}
\text { between parallel plates, } \\
\text { in a rectangular pipe }(w \gtrsim h),
\end{array}\right.\end{cases}
$$

correspondingly, the paraxial approximation produces inaccuracies of $\delta_{1}$ and $\delta_{2}$.

To obtain the impedance per unit length, we divide Eq. (A1) by the circumference of the ring; finally, substitution of the cross products of Eqs. (A20) gives

$$
\frac{Z(k \rho, \beta k)}{Z_{0}}=-\frac{2 \pi i}{\beta h}\left(\frac{2}{k \rho}\right)^{1 / 3} \sum_{p=0}^{\infty} \Lambda_{p}\left(\check{G}_{x}+\beta_{p}^{2} \check{G}_{y}\right) .
$$

Thus we can obtain our CSR impedance for rectangular pipe also from Warnock-Morton's impedance. Other impedances, pillbox chamber and infinite parallel plates, can be obtained in a similar manner. The Airy function $\mathrm{Ci}=$ $\mathrm{Ai}-i \mathrm{Bi}$ corresponds to the Hankel function $H_{n}^{(1)}=J_{n}+$ $i Y_{n}$ in their formalism. Comparing with Eq. (A1), Eq. (A24) has the single variable $k$ and is free of high order Bessel functions. However, the application range is limited in $k \gg \pi / h$, i.e., the longitudinal wave number must be much larger than the waveguide cutoff. In other words, if a bunch length is similar to the chamber height, the paraxial approximation will break down, while Eq. (A1) is right for any wave number.

\section{APPENDIX B: EXPANSION OF THE IMPEDANCE IN THE LOW FREQUENCY LIMIT}

To obtain the impedance in the low frequency limit, we need to expand the cross products of the Airy functions, $\breve{s}(x, y)$ and $\breve{p}(x, y)$, in two steps: (i) the asymptotic series expansion of the Airy functions for large arguments, given by 10.4.59-10.4.67 of [14]; (ii) Taylor series expansion for every portion in the asymptotic expressions. In the low frequency limit, the cross products $\breve{p}\left(v_{p}, u_{p}\right)$ and $\breve{s}\left(v_{p}, u_{p}\right)$ are expanded as follows. Applying the asymptotic expansion of the Airy functions, they are given by

$$
\begin{aligned}
& \check{p}\left(v_{p}, u_{p}\right)=\frac{\left(u_{p} v_{p}\right)^{-1 / 4}}{2 \pi}\left(S_{1} e^{\zeta_{u}-\zeta_{v}}-S_{2} e^{\zeta_{v}-\zeta_{u}}\right), \\
& \check{s}\left(v_{p}, u_{p}\right)=-\frac{\left(u_{p} v_{p}\right)^{1 / 4}}{2 \pi}\left(S_{3} e^{\zeta_{u}-\zeta_{v}}-S_{4} e^{\zeta_{v}-\zeta_{u}}\right) .
\end{aligned}
$$

$u_{p}$ and $v_{p}$ are defined in Eqs. (93) and (94). $\zeta_{u}$ and $\zeta_{v}$ are

$$
\zeta_{u}=(2 / 3) u_{p}^{3 / 2}, \quad \zeta_{v}=(2 / 3) v_{p}^{3 / 2}
$$

$S_{1}, S_{2}, S_{3}$, and $S_{4}$ are the following sums:

$$
\begin{aligned}
& S_{1}=\sum_{m=0}^{\infty} \sum_{n=0}^{\infty}(-1)^{n} \frac{c_{m} c_{n}}{\zeta_{u}^{m} \zeta_{v}^{n}}, \\
& S_{2}=\sum_{m=0}^{\infty} \sum_{n=0}^{\infty}(-1)^{m} \frac{c_{m} c_{n}}{\zeta_{u}^{m} \zeta_{v}^{n}}, \\
& S_{3}=\sum_{m=0}^{\infty} \sum_{n=0}^{\infty}(-1)^{n} \frac{d_{m} d_{n}}{\zeta_{u}^{m} \zeta_{v}^{n}}, \\
& S_{4}=\sum_{m=0}^{\infty} \sum_{n=0}^{\infty}(-1)^{m} \frac{d_{m} d_{n}}{\zeta_{u}^{m} \zeta_{v}^{n}} .
\end{aligned}
$$

The expansion coefficients $c_{n}, d_{n}$ are

$$
c_{n}=\frac{\Gamma(3 n+1 / 2)}{54^{n} n ! \Gamma(n+1 / 2)}, \quad d_{n}=-\frac{6 n+1}{6 n-1} c_{n} .
$$

They have the following relations, respectively:

$$
c_{1}^{2}+c_{1}=2 c_{2}, \quad d_{1}^{2}+d_{1}=2 d_{2} .
$$

We define $\tau$ as

$$
\tau=\frac{1}{4 \beta_{p}^{3}} \simeq \frac{1}{2 \pi}\left(\frac{k}{k_{\mathrm{th}}}\right)^{2},
$$

which is much smaller than one at low frequency: $k<k_{\mathrm{th}}$. $\beta_{p}$ is the vertical wave number of Eq. (26). We expand all equations from Eqs. (B2) to (B3) and also $\left(u_{p} v_{p}\right)^{ \pm 1 / 4}$ of Eqs. (B1) in Taylor series to the second order in $\tau$. For exponential factors in Eqs. (B1), as the zeroth order terms cancel out on the power, it is necessary to expand both $\zeta_{u}$ and $\zeta_{v}$ to the third order with respect to $\tau$ :

$$
\begin{aligned}
& 6 \tau \zeta_{u}=1-6 \tau \hat{x}_{a}+6\left(\tau \hat{x}_{a}\right)^{2}+4\left(\tau \hat{x}_{a}\right)^{3}, \\
& 6 \tau \zeta_{v}=1-6 \tau \hat{x}_{b}+6\left(\tau \hat{x}_{b}\right)^{2}+4\left(\tau \hat{x}_{b}\right)^{3} .
\end{aligned}
$$

$\hat{x}_{a}$ and $\hat{x}_{b}$ are parameters related to the position of the inner and outer walls:

$$
\begin{aligned}
& \hat{x}_{a}=\alpha_{p}\left(x_{a}-\frac{\rho}{2 \gamma^{2}}\right), \\
& \hat{x}_{b}=\alpha_{p}\left(x_{b}-\frac{\rho}{2 \gamma^{2}}\right) .
\end{aligned}
$$

Equations (B3) are expanded to the second order as

$$
\begin{aligned}
& S_{1}=1+\frac{5 \tau^{2}}{2}\left(\hat{x}_{a}-\hat{x}_{b}+1\right), \\
& S_{2}=1+\frac{5 \tau^{2}}{2}\left(\hat{x}_{b}-\hat{x}_{a}+1\right), \\
& S_{3}=1-\frac{7 \tau^{2}}{2}\left(\hat{x}_{a}-\hat{x}_{b}+1\right), \\
& S_{4}=1-\frac{7 \tau^{2}}{2}\left(\hat{x}_{b}-\hat{x}_{a}+1\right) .
\end{aligned}
$$

$\left(u_{p} v_{p}\right)^{ \pm 1 / 4}$ in Eqs. (B1) are given by 
$\frac{\beta_{p}}{\left(u_{p} v_{p}\right)^{1 / 4}}=1+\tau\left(\hat{x}_{a}+\hat{x}_{b}\right)+\tau^{2}\left\{\frac{5}{2}\left(\hat{x}_{a}+\hat{x}_{b}\right)^{2}-4 \hat{x}_{a} \hat{x}_{b}\right\}$,

$\frac{\left(u_{p} v_{p}\right)^{1 / 4}}{\beta_{p}}=1-\tau\left(\hat{x}_{a}+\hat{x}_{b}\right)-\tau^{2}\left\{\frac{3}{2}\left(\hat{x}_{a}+\hat{x}_{b}\right)^{2}-4 \hat{x}_{a} \hat{x}_{b}\right\}$.

Then the cross products in the denominators of the impedance are expanded as follows:

$$
\begin{aligned}
\pi \beta_{p} \check{p}\left(v_{p}, u_{p}\right) & =A_{1} \sinh \left(\hat{x}_{b}-\hat{x}_{a}\right)+A_{2} \cosh \left(\hat{x}_{b}-\hat{x}_{a}\right), \\
-\frac{\pi}{\beta_{p}} \check{s}\left(v_{p}, u_{p}\right) & =B_{1} \sinh \left(\hat{x}_{b}-\hat{x}_{a}\right)+B_{2} \cosh \left(\hat{x}_{b}-\hat{x}_{a}\right),
\end{aligned}
$$

where $A_{1}, A_{2}, B_{1}$, and $B_{2}$ are given by

$$
\begin{aligned}
A_{1}= & +\tau\left(\hat{x}_{a}+\hat{x}_{b}\right)+\frac{\tau^{2}}{2}\left\{\left(\hat{x}_{a}^{2}-\hat{x}_{b}^{2}\right)^{2}\right. \\
& \left.-8 \hat{x}_{a} \hat{x}_{b}+5\left(\hat{x}_{a}+\hat{x}_{b}\right)^{2}+5\right\}, \quad \text { (B16a) } \\
A_{2}= & \tau\left(\hat{x}_{a}^{2}-\hat{x}_{b}^{2}\right)+\frac{\tau^{2}}{2}\left\{\frac{4}{3}\left(\hat{x}_{a}^{3}-\hat{x}_{b}^{3}\right)+2\left(\hat{x}_{a}+\hat{x}_{b}\right)\left(\hat{x}_{a}^{2}-\hat{x}_{b}^{2}\right)\right. \\
& \left.+5\left(\hat{x}_{a}-\hat{x}_{b}\right)\right\}, \quad(\mathrm{B} 16 \mathrm{~b}) \\
B_{1}= & 1-\tau\left(\hat{x}_{a}+\hat{x}_{b}\right)+\frac{\tau^{2}}{2}\left\{\left(\hat{x}_{a}^{2}-\hat{x}_{b}^{2}\right)^{2}+8 \hat{x}_{a} \hat{x}_{b}\right. \\
& \left.-3\left(\hat{x}_{a}+\hat{x}_{b}\right)^{2}-7\right\}, \\
B_{2}= & \tau\left(\hat{x}_{a}^{2}-\hat{x}_{b}^{2}\right)+\frac{\tau^{2}}{2}\left\{\frac{4}{3}\left(\hat{x}_{a}^{3}-\hat{x}_{b}^{3}\right)-2\left(\hat{x}_{a}+\hat{x}_{b}\right)\left(\hat{x}_{a}^{2}-\hat{x}_{b}^{2}\right)\right. \\
& \left.-7\left(\hat{x}_{a}-\hat{x}_{b}\right)\right\} .
\end{aligned}
$$

Then we take terms to the order of $\gamma^{-2}$ in Eqs. (B16). By putting $x_{a}=0$ or $x_{b}=0$ in Eqs. (B9), we can obtain all cross products, e.g., $\check{p}\left(w_{p}, u_{p}\right), \check{s}\left(v_{p}, w_{p}\right)$, which are parts of the impedance. If the sidewalls are symmetric with respect to the beam, i.e., $x_{a}+x_{b}=0$, the summand of Eq. (69) is expanded as

$$
\check{G}_{x}+\beta_{p}^{2} \check{G}_{y}=\frac{\alpha_{p} \tau}{\pi \kappa}\left[3 \tau S\left(\alpha_{p} w\right)-\frac{\alpha_{p} \rho}{\gamma^{2}} T\left(\alpha_{p} w\right)\right] .
$$

The functions $S(x), T(x)$ are given by Eqs. (85) and (86). We thus derived the asymptotic expression of the impedance in the low frequency limit, which is given by Eq. (84). As we mentioned, however, since our theory is based on the paraxial approximation, the asymptotic form is valid in the region: $\pi / h \ll k<k_{\mathrm{th}}$.

\section{APPENDIX C: MULTIVALUE PROBLEM IN THE IMPEDANCE}

Function $\kappa$ has a unique value for positive real wave numbers, but it is multivalued for imaginary wave numbers and negative real ones. In this section we will show that the impedance of Eq. (69) does not depend on the choice for $\kappa$, but it is unique for any real wave numbers and purely imaginary numbers. For a multivalued number, $c=$ $(-1)^{1 / 3}$, if we select $c=-1$, all arguments of the Airy functions $u_{p}, v_{p}$, and $w_{p}$ do not change as $k$ goes to $i k$, so this case is trivial. Next we denote $e^{i \pi / 3}$ as $\varsigma$ :

$$
\varsigma \equiv e^{i \pi / 3}
$$

and consider the values $c=\varsigma^{ \pm 1}$. For the rotation of $k \rightarrow i k$ in the complex wave number plane, the variables of the Airy functions change as follows:

$$
\begin{aligned}
& u_{p}(i k)=\varsigma^{\mp 2} \bar{u}_{p}(k), \\
& v_{p}(i k)=\varsigma^{\mp 2} \bar{v}_{p}(k), \\
& w_{p}(i k)=\varsigma^{\mp 2} \bar{w}_{p}(k) .
\end{aligned}
$$

We apply identities of the Airy functions, 10.4.6-10.4.9 of [14]:

$$
\begin{aligned}
\operatorname{Ai}\left(s^{ \pm 2} z\right) & =\frac{s^{ \pm 1}}{2}[\operatorname{Ai}(z) \mp i \operatorname{Bi}(z)], \\
\operatorname{Bi}\left(s^{ \pm 2} z\right) & =\frac{s^{ \pm 1}}{2}[\operatorname{Bi}(z) \mp 3 i \operatorname{Ai}(z)], \\
\operatorname{Ai}^{\prime}\left(s^{ \pm 2} z\right) & =\frac{s^{\mp 1}}{2}\left[\operatorname{Ai}^{\prime}(z) \mp i \operatorname{Bi}^{\prime}(z)\right], \\
\operatorname{Bi}^{\prime}\left(s^{ \pm 2} z\right) & =\frac{s^{\mp 1}}{2}\left[\operatorname{Bi}^{\prime}(z) \mp 3 i \operatorname{Ai}^{\prime}(z)\right],
\end{aligned}
$$

where $z$ is an arbitrary complex variable, and the primes denote the derivatives with respect to the argument. By these identities, the cross products change as

$$
\begin{aligned}
& \check{p}\left(\varsigma^{ \pm 2} x, \varsigma^{ \pm 2} y\right)=\varsigma^{ \pm 2} \check{p}(x, y), \\
& \check{q}\left(\varsigma^{ \pm 2} x, \varsigma^{ \pm 2} y\right)=\check{q}(x, y), \\
& \check{r}\left(\varsigma^{ \pm 2} x, \varsigma^{ \pm 2} u\right)=\check{r}(x, y), \\
& \check{s}\left(\varsigma^{ \pm 2} x, \varsigma^{ \pm 2} y\right)=\varsigma^{\mp 2} \check{s}(x, y) .
\end{aligned}
$$

Using Eqs. (C6), one can show that the following relations hold for any value of $c$ :

$$
R_{j}(i k)+\bar{R}_{j}(k)=0
$$

where $R_{j}, \bar{R}_{j}(j=x, y)$ are given by Eqs. (143) and (149), respectively. We define $P_{x}$ and $P_{y}$ as 


$$
\begin{aligned}
& P_{x}(k)=\frac{\kappa}{k} \frac{\check{s}\left(v_{p}, w_{p}\right) \check{s}\left(w_{p}, u_{p}\right)}{\check{s}\left(v_{p}, u_{p}\right)}, \\
& P_{y}(k)=\beta_{p}^{2} \frac{\kappa}{k} \frac{\check{p}\left(v_{p}, w_{p}\right) \check{p}\left(w_{p}, u_{p}\right)}{\check{p}\left(v_{p}, u_{p}\right)} .
\end{aligned}
$$

$P_{x}+P_{y}$ is the imaginary impedance of Eq. (69) omitting the coefficient. Similarly, we define $\bar{P}_{x}$ and $\bar{P}_{y}$ as

$$
\begin{aligned}
& \bar{P}_{x}(k)=\frac{\kappa}{k} \frac{\check{s}\left(\bar{v}_{p}, \bar{w}_{p}\right) \check{s}\left(\bar{w}_{p}, \bar{u}_{p}\right)}{\check{s}\left(\bar{v}_{p}, \bar{u}_{p}\right)}, \\
& \bar{P}_{y}(k)=\beta_{p}^{2} \frac{\kappa}{k} \frac{\check{p}\left(\bar{v}_{p}, \bar{w}_{p}\right) \check{p}\left(\bar{w}_{p}, \bar{u}_{p}\right)}{\check{p}\left(\bar{v}_{p}, \bar{u}_{p}\right)} .
\end{aligned}
$$

From Eqs. (C6), it is shown for any value of $c$ that

$$
P_{j}(i k)=i \bar{P}_{j}(k)
$$

where $j=x, y$. If $x_{a}+x_{b}=0$ and $\gamma=\infty$, the variable of the Airy functions have special relations as

$$
\begin{aligned}
& u_{p}(i k)=c^{-2} v_{p}(k), \\
& v_{p}(i k)=c^{-2} u_{p}(k), \\
& w_{p}(i k)=c^{-2} w_{p}(k) .
\end{aligned}
$$

Then the following relations hold:

$$
\begin{aligned}
& R_{j}(k)+\bar{R}_{j}(k)=0, \\
& P_{j}(k)+\bar{P}_{j}(k)=0 .
\end{aligned}
$$

In this case the impedance of a rectangular pipe has a symmetry between the real axis and the imaginary axis:

$$
Z( \pm i k)=\mp i Z(k) .
$$

For negative real wave numbers, the variable $\kappa$ becomes

$$
\kappa(-k)=c^{2} \kappa(k) .
$$

Then the variables of the impedance change as follows:

$$
\begin{aligned}
& u_{p}(-k)=c^{2} u_{p}(k), \\
& v_{p}(-k)=c^{2} v_{p}(k), \\
& w_{p}(-k)=c^{2} w_{p}(k), \\
& \beta_{p}^{2}(-k)=c^{2} \beta_{p}^{2}(k) .
\end{aligned}
$$

For $c=-1$, it is merely identity and trivial. For other values, $c=\mathrm{s}^{ \pm 1}$, the variables can be treated in the same manner as the imaginary wave numbers. Then one finds

$$
\begin{gathered}
R_{j}(-k)=+R_{j}(k), \\
P_{j}(-k)=-P_{j}(k), \\
Z(-k)=Z^{*}(k) .
\end{gathered}
$$

In the paraxial approximation, thus the CSR impedance of a rectangular pipe has a unique value for any wave number on the real axis and the imaginary axis (while the parallel plates impedance depends on the branch $n_{b} \in \mathbb{Z}$ in $\arg k+$ $\left.2 \pi n_{b}\right)$. We used the symmetries of $P_{j}(k), \bar{P}_{j}(k)$ and $R_{j}(k)$, $\bar{R}_{j}(k)$ to investigate the pole structure in Sec. IV and also to rearrange the integral of Eq. (147).

[1] J. Schwinger (unpublished).

[2] R. L. Warnock and P. Morton, Part. Accel. 25, 113 (1990); SLAC Report No. SLAC-PUB-4562, 1988.

[3] K. Y. Ng, Part. Accel. 25, 153 (1990).

[4] R.L. Warnock, SLAC Report No. SLAC-PUB-5375, 1990.

[5] J. B. Murphy, S. Krinsky, and R. L. Gluckstern, Part. Accel. 57, 9 (1997).

[6] Ya. S. Derbenev, J. Rossbach, E.L. Saldin, and V.D. Shiltsev, DESY Report No. TESLA-FEL 95-05, 1995.

[7] Ya.S. Derbenev and V.D. Shiltsev, SLAC Report No. SLAC-PUB-7181, 1996.

[8] E. L. Saldin, E. A. Schneidmiller, and M. V. Yurkov, Nucl. Instrum. Methods Phys. Res., Sect. A 398, 373 (1997).

[9] C. Mayes and G. Hoffstaetter, Phys. Rev. ST Accel. Beams 12, 024401 (2009), http://arxiv.org/abs/0812.3189v1.

[10] G. V. Stupakov and I. A. Kotelnikov, Phys. Rev. ST Accel. Beams 6, 034401 (2003).

[11] T. Agoh and K. Yokoya, Phys. Rev. ST Accel. Beams 7, 054403 (2004).

[12] T. Agoh, Ph.D. thesis, University of Tokyo, 2004, http:// acc-physics.kek.jp/Ago/Agoh_thesis.pdf.

[13] ICFA Beam Dynamics Newsletter, No. 35, edited by C. Biscari (ICFA Beam Dynamics Panel, Batavia, Illinois, 2004).

[14] Handbook of Mathematical Functions, edited by M. Abramowitz and I. A. Stegun (Dover, New York, 1972).

[15] D. C. Sagan, G. H. Hoffstaetter, C. E. Mayes, and U. SaeUeng, arXiv:0806.2893v1, http://arxiv.org/abs/0806. $2893 \mathrm{v} 1$

[16] K. Y. Ng and R. Warnock, Phys. Rev. D 40, 231 (1989).

[17] Wolfram Research Inc., The Wolfram Functions Site, http://functions.wolfram.com/.

[18] A. W. Chao, Physics of Collective Beam Instabilities in High Energy Accelerators (Wiley, New York, 1993).

[19] K. Yokoya, Part. Accel. 41, 221 (1993).

[20] M. Lax, W. H. Louisell, and W. B. McKnight, Phys. Rev. A 11, 1365 (1975). 